\title{
Role of Uric Acid Metabolism-Related Inflammation in the Pathogenesis of Metabolic Syndrome Components Such as Atherosclerosis and Nonalcoholic Steatohepatitis
}

\author{
Akifumi Kushiyama, ${ }^{1}$ Yusuke Nakatsu, ${ }^{2}$ Yasuka Matsunaga, ${ }^{2}$ \\ Takeshi Yamamotoya, ${ }^{2}$ Keiichi Mori, ${ }^{2}$ Koji Ueda, ${ }^{2}$ Yuki Inoue, ${ }^{2}$ Hideyuki Sakoda, ${ }^{3}$ \\ Midori Fujishiro, ${ }^{4}$ Hiraku Ono, ${ }^{5}$ and Tomoichiro Asano ${ }^{2}$ \\ ${ }^{1}$ Division of Diabetes and Metabolism, Institute for Adult Disease, Asahi Life Foundation, 1-6-1 Marunouchi, \\ Chiyoda-ku, Tokyo, Japan \\ ${ }^{2}$ Department of Medical Science, Graduate School of Medicine, Hiroshima University, 1-2-3 Kasumi, \\ Minami-ku, Hiroshima City, Hiroshima, Japan \\ ${ }^{3}$ Division of Neurology, Respirology, Endocrinology and Metabolism, Department of Internal Medicine, Faculty of Medicine, \\ University of Miyazaki, Miyazaki 889-1692, Japan \\ ${ }^{4}$ Department of Internal Medicine, Graduate School of Medicine, University of Tokyo, 7-3-1 Hongo, Bunkyo-ku, Tokyo, Japan \\ ${ }^{5}$ Department of Endocrinology and Diabetes, School of Medicine, Saitama Medical University, Moroyama, Saitama 350-0495, Japan
}

Correspondence should be addressed to Akifumi Kushiyama; kusiyaa-tky@umin.ac.jp

Received 18 September 2016; Revised 3 November 2016; Accepted 15 November 2016

Academic Editor: Jie Yin

Copyright (C) 2016 Akifumi Kushiyama et al. This is an open access article distributed under the Creative Commons Attribution License, which permits unrestricted use, distribution, and reproduction in any medium, provided the original work is properly cited.

\begin{abstract}
Uric acid (UA) is the end product of purine metabolism and can reportedly act as an antioxidant. However, recently, numerous clinical and basic research approaches have revealed close associations of hyperuricemia with several disorders, particularly those comprising the metabolic syndrome. In this review, we first outline the two molecular mechanisms underlying inflammation occurrence in relation to UA metabolism; one is inflammasome activation by UA crystallization and the other involves superoxide free radicals generated by xanthine oxidase (XO). Importantly, recent studies have demonstrated the therapeutic or preventive effects of $\mathrm{XO}$ inhibitors against atherosclerosis and nonalcoholic steatohepatitis, which were not previously considered to be related, at least not directly, to hyperuricemia. Such beneficial effects of XO inhibitors have been reported for other organs including the kidneys and the heart. Thus, a major portion of this review focuses on the relationships between UA metabolism and the development of atherosclerosis, nonalcoholic steatohepatitis, and related disorders. Although further studies are necessary, XO inhibitors are a potentially novel strategy for reducing the risk of many forms of organ failure characteristic of the metabolic syndrome.
\end{abstract}

\section{Introduction}

Uric acid (UA) is the end product of the metabolic pathway for purines, the main constituents of nucleotides. The pathway of UA generation is shown in Figure 1. Briefly, inosine monophosphate (IMP) is derived from de novo purine synthesis and from purine salvage. Hypoxanthine from IMP is catalyzed to xanthine and then to uric acid by xanthine oxidase (XO). De novo nucleotide synthesis generates IMP via ribose-5-phosphate, catalyzed to 5-phosphoribosyl-1-pyrophosphate (PRPP). In the salvage pathway, hypoxanthine-guanine phosphoribosyl transferase (HGPRT) plays an important role in generating IMP, thereby inhibiting UA generation.

Since humans are unable to catabolize UA to the more soluble compound allantoin due to lack of urate oxidase or uricase [1], the serum UA concentration is higher in humans than almost all other mammals. However, this high UA level in humans has been regarded as being beneficial in the presence of elevated oxidative stress [2]. UA is oxidized to allantoin and other metabolites via nonenzymatic oxidation 


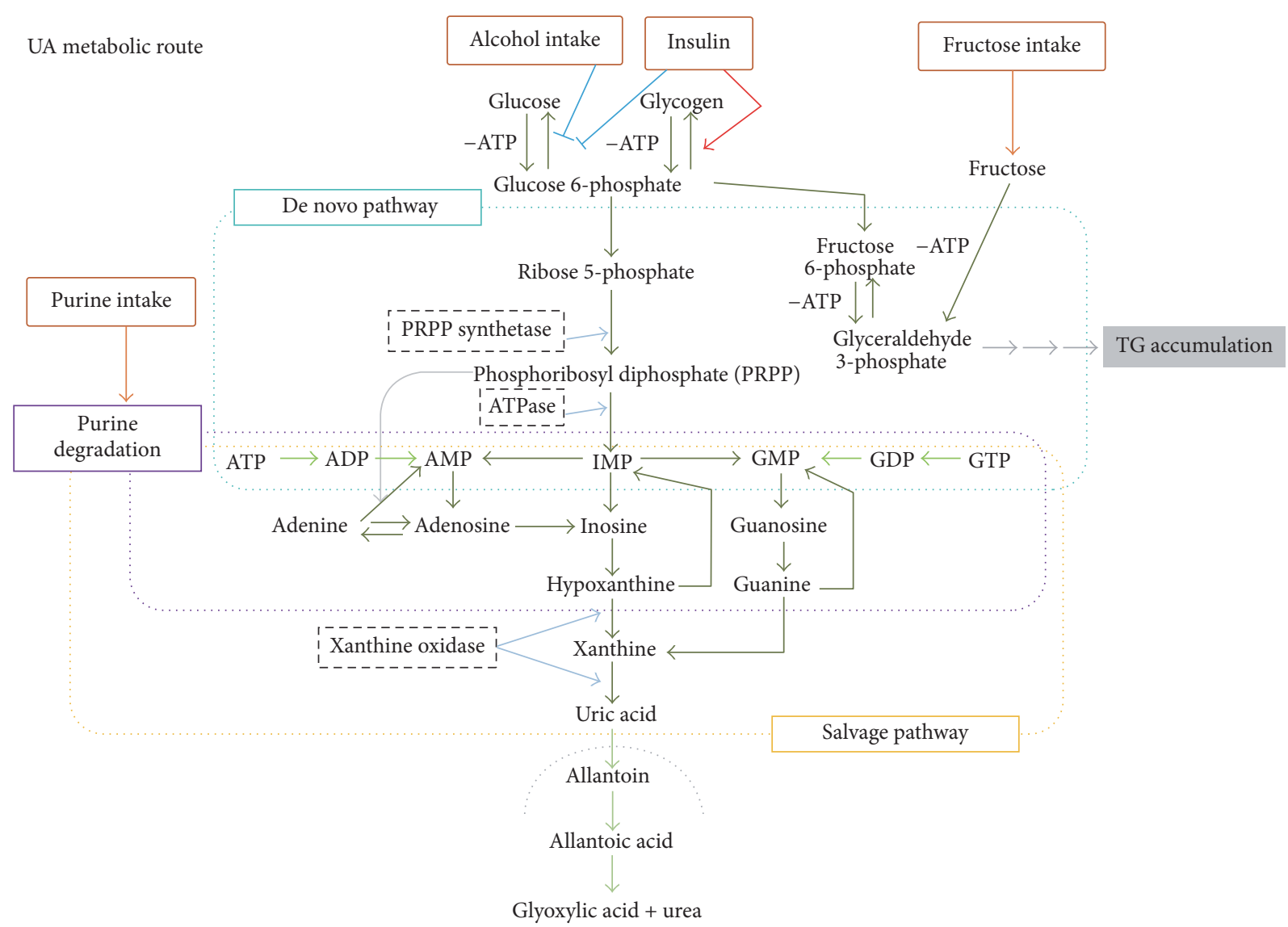

FIgURE 1: Metabolic pathways involving UA.

[3] and, thus, UA can function to neutralize prooxidant molecules, such as hydroxyl radicals, hydrogen peroxide, and peroxynitrite. UA shows the highest scavenging rate constant against $\mathrm{O}_{2}^{-}$, with constants being low against $\mathrm{CH}^{\circ}$ and $\mathrm{t}^{\circ} \mathrm{BuOO}^{\circ}$ [4]. UA directly (nonenzymatically) and preferentially deletes nitric oxide (NO) and forms 6aminouracil in physiological environments or in association with antioxidants [5]. In vitro, UA has both an antioxidant effect on native LDL and a prooxidant effect on mildly oxidized LDL [6]. Allantoin does not have these effects. The mechanisms of these reactions vary among combinations of prooxidant molecules and solution polarities [7].

It has been suggested that this antioxidant effect of the high UA concentrations in humans contributes to neuroprotection in several neurodegenerative and neuroinflammatory diseases [8-14].

However, despite the potential antioxidant effect of UA itself, numerous studies have revealed close associations of serum UA concentrations and various disorders, most of which are included in the metabolic syndrome category. Thus, UA metabolism may be a so-called double-edged sword as regards the inflammatory and/or oxidative responses in many organs, though on the whole, its harmful effects appear to outweigh the benefits of UA in most cases.

In this review, we first explain the two putative molecular mechanisms underlying inflammation occurrence in relation to UA metabolism; one is inflammasome activation via UA crystallization and the other involves superoxide free radicals generated by XO. While the UA crystallization mechanism would be dependent on a high serum UA concentration, the latter may not necessarily reflect the serum UA concentration though $\mathrm{XO}$ activity does lead to the production of reactive oxygen species (ROS).

Subsequently, lines of research showing relationships between UA metabolism and the development of various disorders are introduced and discussed. Importantly, recent studies have demonstrated beneficial effects of XO inhibitors against the occurrence and/or progression of several disorders, particularly atherosclerosis and nonalcoholic steatohepatitis (NASH), both of which are associated with insulin resistance, hyperlipidemia, and/or obesity. In this review, atherosclerosis and NASH are discussed extensively, while studies of gout and chronic kidney diseases (CKD) are mentioned briefly. In conclusion, we propose that such $\mathrm{XO}$ inhibitors may be more useful for preventing a variety of disorders, such as atherosclerosis and NASH, than previously believed, probably via an anti-inflammatory effect.

\section{Inflammation Occurrence Related to UA Metabolism}

Among the disorders related to hyperuricemia, gout is the most representative and well known. Features of gout include painful arthritis affecting the limbs, caused by reduced UA 
crystals in the joints. While symptoms of a gout attack are typical of an acute inflammatory response, as indicated by the presence of swelling, heat, rubescence, and pain, there are many disorders with mild but chronic inflammation which are very likely to be related to UA metabolism. In the latter case, superoxide free radicals generated by XO are key players leading to chronic inflammatory processes eventually resulting in impaired organ functions. Thus, we introduce two independent mechanisms underlying UA metabolisminduced inflammation.

2.1. Inflammasome Activation by Crystallized UA Particles. In 2002, the inflammasome concept was proposed to involve multiple proteins and to control the cleavage of prointerleukin 1 (IL-1) [15]. Initially, inflammasomes were considered to play a role in immune responses and serve as defense systems against pathogens $[16,17]$. However, a line of subsequent studies has elucidated that inflammasomes are key players in the onsets of a wide range of diseases as well as host defense. Excessive metabolites, such as ATP or monosodium urate crystals (MUC), were also confirmed to be involved in the activation of inflammasomes, and inflammatory responses occurring via inflammasomes have been demonstrated to be linked to the onset and progression of human diseases, including gout, atherosclerosis and NASH, as described below in detail [18-24].

Inflammasomes are known to be divided into discernible patterns, depending on component proteins [16]. Among them, the NLRP3 inflammasome, comprised of three major components, Nod-like receptor 3 (NLRP3), apoptosisassociated speck-like protein containing a CARD (ASC) and caspase-1, has been well investigated. Maturations of both IL-1 and IL-18 by inflammasomes require a two-step mechanism. First, the Toll-like receptor ligands, such as lipopolysaccharide (LPS), activate the NF- $\kappa \mathrm{B}$ pathway and upregulate the expression levels of interleukins, including pro-IL- $1 \beta$ and pro-IL-18. Subsequently, the inflammasome complex activated by pathogen-associated molecular patterns (PAMPs) or damage-associated molecular patterns (DAMPs) cleaves pro-IL-1 $\beta$ or pro-IL-18, resulting in the production of mature interleukins [15-17].

MUC also reportedly serve as a danger signal and trigger the activation of inflammasomes [18]. Although the mechanism of inflammasome activation by MUC has not been fully elucidated, the following mechanism was proposed. MUC stimulate the Toll-like receptor $2 / 4-M y d 88$ pathway and raise transcriptional levels of pro-IL-1 $\beta$ through the $\mathrm{NF}-\kappa \mathrm{B}$ pathway [25]. It is theorized that MUC-induced inflammasome activation is driven by two key factors. One is a decrease in the intracellular potassium concentration. Indeed, the addition of high potassium abrogated IL-1 $\beta$ release by MUC. The other is the generation of ROS, because an antioxidant, $\mathrm{N}$-acetyl-cysteine, abolished IL- $1 \beta$ secretion by MUC [26]. Other studies have indicated the application of MUC to raise intracellular ROS levels. However, the relationship between intracellular $\mathrm{K}^{+}$level changes and ROS generation remains unknown, and future studies are expected to resolve this issue $[27,28]$. Elevation of intracellular ROS mediates the detachment of thioredoxin-interacting protein
(TXNIP) from thioredoxin and enables TXNIP to associate with NLRP3, leading to NLRP3 inflammasome activation $[29,30]$. Thus, MUC accumulation promotes inflammatory responses through inflammasomes (Figure 2) and thereby promotes the onset of diseases, such as gout.

2.2. Superoxide Free Radicals Generated by XO. When mammalian xanthine dehydrogenase $(\mathrm{XDH})$ is converted to $\mathrm{XO}$ under stressed conditions such as tissue damage and ischemia [31], superoxide anion and hydrogen peroxide are produced during molybdenum hydroxylase-catalyzed reactions in a molar ratio of about 1:3 [32]. The proteolytic activation from $\mathrm{XDH}$ to $\mathrm{XO}$ is required for superoxide generation [33]. In essence, $\mathrm{XO}$ oxidizes a variety of purines and pterins, classified as molybdenum iron-sulfur flavin hydroxylases. When $\mathrm{XO}$ reacts with xanthine, electrons are transferred from Mo, Fe-S, and FAD. XO produces FADH2, while XDH produces FADH. Only FADH2 reacts with $\mathrm{O} 2$ [34]. In the UA metabolic pathway, XO oxidizes hypoxanthine from nucleic acid metabolites into xanthine and xanthine into UA (Figure 1). XO, as well as nicotinamide adenine dinucleotide phosphate (NADPH) oxidase and the mitochondrial electron-transport chain, generates ROS [35].

ROS from XO might play physiological roles, especially in development. Treatment during pregnancy with allopurinol alters maternal vascular function involving $\beta 1$-adrenergic stimulation and impairs the fetal $\alpha 1$-adrenergic vasoreflex response involving NO [36]. Fetal XO is activated in vivo during hypoxia and $\mathrm{XO}$-derived ROS contributes to fetal peripheral vasoconstriction, leading to fetal defense against hypoxia [37]. XO depletion induces renal interstitial fibrosis, and renal epithelial cells from XOR (-/-) mice are more readily transformed into myofibroblasts [38]. Indeed, how ROS from XO directly and physiologically acts in vivo is unknown.

The tissue and cellular distributions of XO in mammals are highest in the liver and intestines due to $\mathrm{XO}$-rich parenchymal cells [39]. Xanthine oxidoreductase (XOR) is present in hepatocytes, while $\mathrm{XO}$ is present in bile duct epithelial cells, concentrated toward the luminal surface. Moreover, in human liver disease, proliferating bile ducts are also strongly positive for XO [40]. Molybdenum supplementation significantly increased $\mathrm{XO}$ activities in the liver and small intestinal mucosa [41]. XO activity is low in human serum, the brain, heart, and skeletal muscle, while being rich in microvascular endothelial cells [42] and is also present in macrophages [43]. Circulating XO can adhere to endothelial cells by associating with endothelial glycosaminoglycans [44]. The study using electron spin resonance measurements revealed the contribution of increased $\mathrm{XO}$ activity to endothelial dysfunction in patients with coronary artery diseases [45].

$\mathrm{XO}$ activation is induced by LPS, angiotensin II, NADPH oxidase, hypoxia, hypoxia-inducible factor 1 , and inflammatory cytokines such as IL-1 $\beta$ [46-49]. The release of XO is increased in hypercholesterolemia, chronic hyperammonemia, thermal trauma, beta-thalassemia, brain ischemia, and pulmonary artery hypertension [50-54]. Aging is another factor associated with elevated $\mathrm{XO}$ activity. Indeed, $\mathrm{XO}$ was 


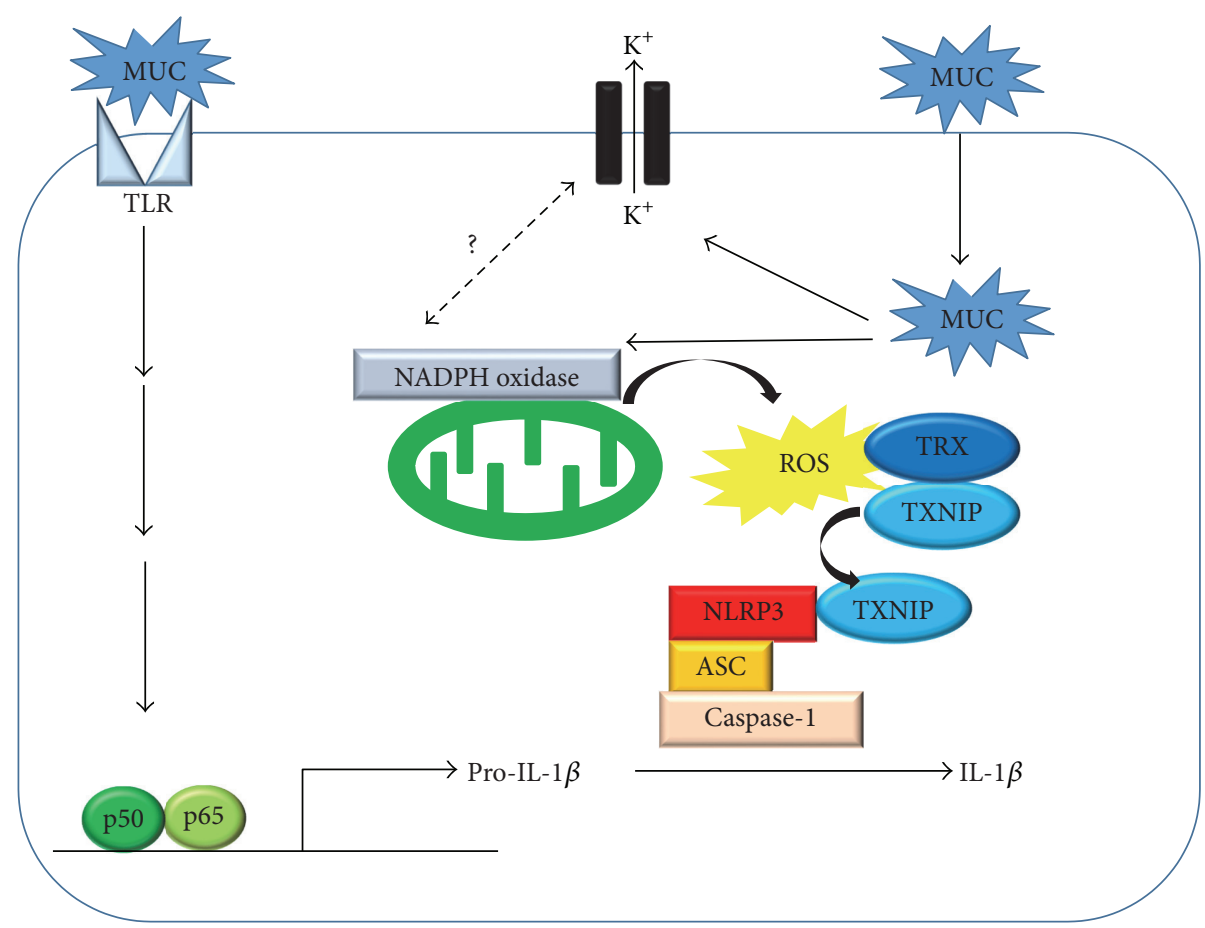

FIGURE 2: MUC induces inflammasome activation. MUC activates the NF- $\kappa$ B pathway through TLR2/4, thereby increasing the expressions of pro-IL-1 $\beta$ or pro-IL-18. At the same time, MUC induces ROS release from mitochondria. The generated ROS detaches TXNIP from thioredoxin and enables TXNIP to interact with the NLRP3 complex. The binding of TXNIP to NLRP3 activates inflammasomes, leading to the production of mature IL-1 $\beta$ or IL-18. MUC: monosodium urate crystals, TLR: Toll-like receptor, TXNIP: thioredoxin-interacting protein, TXR: thioredoxin, and ROS: reactive oxygen species.

significantly higher in the aortic walls and skeletal muscles of old rats than in those of their young counterparts. The correlation between plasma XO activity and age is observed in both humans and rats [55]. It appears that hyperglycemia itself has no impact on liver XO activity, though cardiac, renal, and brain XO activities were shown to be increased in rats with advanced diabetes [56, 57]. XO activity rises remarkably in ischemic congestive heart failure and $\mathrm{XO}$ localizes within CD68 positive macrophages [43]. The association between $\mathrm{XO}$ and ischemic reperfusion injury has been well investigated. $\mathrm{XO}$ is one of the major superoxide sources in ischemia/reperfusion injuries of the heart [58], forebrain [59], skin [60], liver [61, 62], and gastric mucosa [63], as well as multiple system organ failure after hind limb reperfusion [64]. XO activity, along with lipid peroxidation, myeloperoxidase activity and NO levels, is increased in the liver in response to renal ischemia/reperfusion in diabetic rats [65]. Ischemia/reperfusion injury is attributable to elevated $\mathrm{XO}$ activity and ATP depletion related to increasing hypoxanthine and xanthine levels during ischemia, and reperfusion provides $\mathrm{O}_{2}$ for oxidation of these compounds [1].

Superoxide production by XO may also be enhanced by increasing the amount of its substrate, purine bodies. Excess fructose metabolism results in ATP depletion which is associated with degradation of AMP to hypoxanthine, followed by conversion to UA by XO [66]. Indeed, the serum UA level is upregulated in response to a fructose burden [67]. Inversely, UA stimulates fructokinase and fructose metabolism during fatty liver development [68]. ATP depletion, such as that characteristic of glycogen storage disease type 1 [69], hypoglycemia [70], exercise [71], and starvation [72], also increases UA production. Conditions associated with DNA turnover, such as tumor progression [73] and tumor lysis [74], are also mediated by XO.

Superoxide produced by $\mathrm{XO}$ is an important messenger inducing inflammation and signal transduction, leading to tissue damage. We found inflammatory cytokines to be induced via $\mathrm{XO}$ when foam cells form with lipid accumulation [75]. XO regulates cyclooxygenase-2 [76] in the inflammatory system, and XO appears to be critical for innate immune function [77]. XO increased Egr-1 mRNA and protein, as well as the phosphorylation of ERK1/2, while pretreatment with an ERK1/2 inhibitor prevented induction of Egr-1 by XO [78]. In addition, XO reportedly reduced SUMOylation of PPAR $\gamma$ in inflammatory cells [79]. ROS from XO augment TRB3 expression in podocytes [80].

As noted above, superoxide from $\mathrm{XO}$ has been suggested to play roles in various forms of inflammatory or ischemic pathophysiology (Figure 3), not necessarily involving hyperuricemia.

\section{UA Metabolism and Chronic Renal Disease, Atherosclerosis, Heart Failure, and NASH}

While gout is a disorder well known to be caused by the precipitation of UA crystals, the involvement of hyperuricemia in CKD is also widely recognized. The major causes of CKD have been regarded as diabetes mellitus and hypertension, 


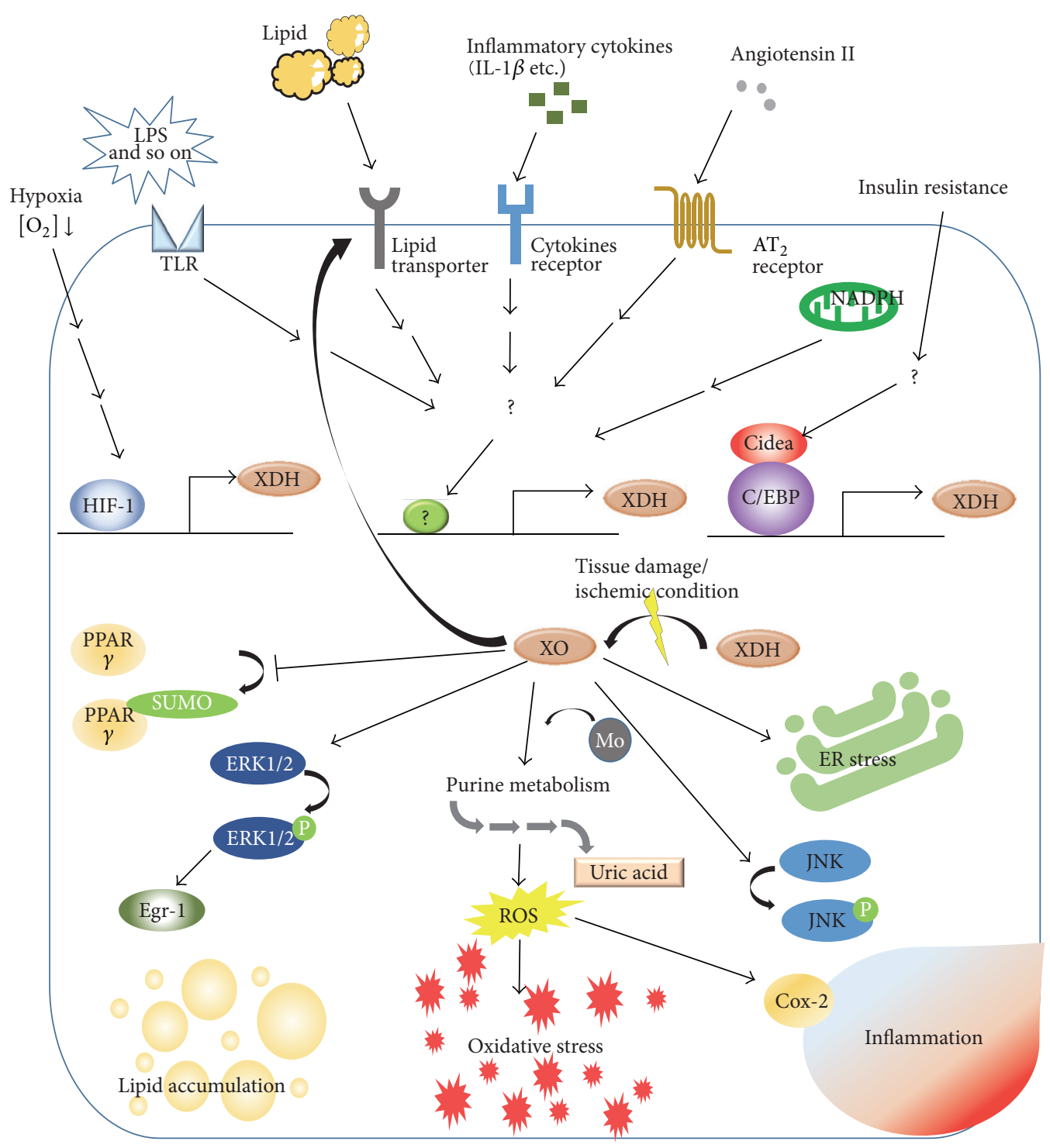

FIGURE 3: Involvement of XO in molecular pathologies related to inflammation; "causes and results."

and thus, hyperuricemia was long viewed as a consequence of CKD. In fact, loss of kidney function reduces the excretion of UA into urine, resulting in hyperuricemia. In contrast, recent studies demonstrated a significant association between serum UA and the development of CKD. While each metabolic syndrome component, including hyperglycemia, hyperlipidemia, and hypertension, was associated with an increased CKD risk, hyperuricemia was apparently an independent risk factor not influenced by the others. Therefore, hyperuricemia is both a cause and a consequence of CKD and is frequently associated with other metabolic syndrome features.

In terms of CKD pathogenesis, serum UA is likely to activate the renin-angiotensin system resulting in vascular smooth muscle cell proliferation [81] and to induce an epithelial-to-mesenchymal transition of renal tubular cells [82]. XO inhibitor treatment reportedly reduced intercellular adhesion molecule-1 (ICAM-1) expression in tubular epithelial cells [83] of mice. We speculate that UA itself and superoxide free radical generation both play roles in the molecular mechanisms underlying hyperuricemiarelated CKD development, but further research is required to elucidate the complex mechanistic interactions between serum UA and CKD.

As mentioned in Section 2, both UA and superoxide free radicals are simultaneously produced by $\mathrm{XO}$ and might be the pathophysiological cause of these diseases. As shown in Figure 3, chronic inflammation is also involved in pathophysiological processes, generally exhibiting a close relationship with oxidative stress. ROS from XO induces LPS-induced JNK activation via inactivation of MAPK phosphatase(MKP-) 1 [84] and XO regulates cyclooxygenase-2, one of the master regulators of inflammation [76]. Therefore, damage from UA, ROS, and UA-induced and/or ROS-induced inflammation might together contribute to the progression of certain diseases, and distinguishing which mechanism acts first is often difficult in lifestyle-related diseases. 
3.1. Atherosclerosis, Vascular Dysfunction, and Heart Failure. Although the relationships between serum UA levels and atherosclerotic diseases, including hypertension $[85,86]$, have been documented, whether or not serum UA itself is an independent cardiovascular risk factor remains controversial as most hyperuricemic patients with cardiovascular diseases (CVD) have other complications such as hypertension, dyslipidemia, diabetes, and CKD as well, which are generally regarded as more established risk factors for CVD than hyperuricemia. Recently, however, a growing body of evidence from both clinical and basic research supports the hypothesis that hyperuricemia, partly via elevated XO activity, is an independent risk factor for hypertension and CVD.

Despite the association between hyperuricemia and hypertension having been recognized since the 19th century [85], it was not until recently that hyperuricemia was demonstrated to be an independent risk factor for hypertension development [87-93]. A recently published metaanalysis showed that the adjusted relative risk of developing hypertension was 1.48 for hyperuricemic patients [94], and this association was apparently much stronger in younger, early-onset hypertensive patients [86, 95]. Several clinical trials have demonstrated the beneficial effects of UA lowering therapy for hypertension [96-99]. In a trial targeting prehypertensive obese adolescents, administration of either allopurinol (XO inhibitor) or probenecid (uricosuric agent) lowered blood pressure [98]. Consistently, both allopurinol and benziodarone (uricosuric agent) reduced blood pressure in rats with hypertension induced by hyperuricemia [100, 101], suggesting that not only XO activity but also UA itself plays an important role in the pathogenesis of hypertension.

Besides the association with hypertension, hyperuricemia or gout has been confirmed to be related to the morbidity and the mortality of CVD [102-106]. According to a recently published meta-analysis [107], the relative risks of morbidity and mortality for coronary heart diseases were 1.13 and 1.27 , respectively, in hyperuricemic patients as compared to controls. Several clinical studies have indicated the benefits of XO inhibitors for reducing the incidence of myocardial infarction [108], improving exercise tolerance in patients with stable angina [109], and enhancing endothelial function $[110,111]$. However, interestingly, unlike the case of treating hypertension, uricosuric agents have failed to show any benefits in patients with hyperuricemia or gout $[110,112]$.

What are the mechanisms underlying the aforementioned association between hyperuricemia and atherosclerotic diseases? First, the role of XO in the pathogenesis of atherosclerosis merits attention. As described above, XO produces ROS when converting hypoxanthine into xanthine and then UA. $\mathrm{XO}$ is also expressed in endothelial cells [113] and was shown to be increased in the aortic endothelial cells of $\mathrm{ApoE}^{-/-}$mice [114], an established model of atherosclerosis. Since oxidative stress inactivates NO and leads to endothelial dysfunction [115], endothelial XO, especially given its enhanced expression during the development of atherosclerosis, contributes to vascular damage via ROS production.

Recently, we established that XO activity in macrophages also plays a key role in the development of atherosclerosis [75]. During atherosclerosis development, monocytes migrate beneath the endothelium and transform into macrophages, which then turn into foam cells by incorporating modified low density lipoproteins (LDL) (such as oxidized LDL and acetyl LDL) or very low density lipoproteins (VLDL). Foam cells contribute to the formation of unstable plaques by secreting inflammatory mediators and matrix-degrading proteases (such as matrix metalloproteinases (MMPs)) and by generating a prothrombotic necrotic core by eventually undergoing necrotic or apoptotic death [116]. We demonstrated that allopurinol treatment ameliorated aortic lipid accumulation and calcification of the vessels of ApoE-KO mice and that allopurinol markedly suppressed the transformation of J774.1 murine macrophages or primary cultured human macrophages into foam cells in response to stimulation with acetyl LDL or VLDL. The expressions of scavenger receptors (SR-A1, SR-B1, and SR-B2) and VLDL receptors in J774.1 cells were upregulated by XOR overexpression and downregulated by siRNA-mediated XOR suppression, raising the possibility that $\mathrm{XO}$ activity in macrophages positively regulates foam cell formation by increasing the uptake of modified LDL or VLDL. Conversely, expressions of ABCA1 and ABCG1, which regulate cellular cholesterol efflux, were decreased by XOR overexpression and increased by XOR knockdown. Furthermore, allopurinol suppressed the expressions of inflammatory cytokines such as IL-1 $\beta$, IL-6, IL12 , and TNF $\alpha$, and the expressions of VCAM1, MCP-1, and MMP2, which were upregulated in J774.1 cells transformed into foam cells by atherosclerogenic serum. Subsequently, febuxostat, another XO inhibitor, was also demonstrated to attenuate the development of atherosclerotic lesions in $\mathrm{ApoE}^{-/-}$mice [114]. That study showed XO expression to be increased in macrophages infiltrating atherosclerotic plaques and that febuxostat diminished the ROS level in the aortic walls of $\mathrm{ApoE}^{-/-}$mice. The authors demonstrated that cholesterol crystals (CCs) increased endogenous XO activity and ROS production in macrophages and that CCs enhanced not only IL-1 $\beta$ release via NLRP3 inflammasome activation but also secretions of other inflammatory cytokines such as IL-1 $\alpha$, IL-6, and MCP-1 from macrophages, processes which in turn were suppressed by febuxostat or ROS inhibitors. The significance of NLRP3 inflammasome activation in macrophages by CCs was verified by the observation that atherosclerosis in high-cholesterol diet fed LDL receptor(LDLR-) deficient mice was alleviated by transplanting bone marrow from NLRP3-deficient, ASC-deficient, or IL- $1 \alpha / \beta$ deficient mice [117]. Taking these observations together, we can reasonably speculate that XO in macrophages enhances foam cell formation, ROS production, and NLRP3 inflammasome activation, all three of which exacerbate inflammation and plaque formation, thereby contributing to the development of atherosclerotic diseases [75, 114-116].

Independently of $\mathrm{XO}$, UA itself is widely recognized to exert direct effects on vascular functions. Vascular endothelial cells express several UA transporters [118] and incorporated UA impairs NO production and leads to endothelial dysfunction $[118,119]$. In vascular smooth muscle cells, UA stimulates proliferation and ROS production and inhibits NO production via increased angiotensin II expression $[81,120]$. As noted above, not only $\mathrm{XO}$ inhibitors but also uricosuric 
agents markedly lowered blood pressure, especially in studies targeting early-stage hypertensive patients [98] and those using animal models $[100,101]$. The results obtained suggest that UA presumably contributes to early-stage hypertension by promoting renal vasoconstriction via reduced NO production and activation of the renin-angiotensin system [86, 98].

3.2. Nonalcoholic Steatohepatitis. The number of nonalcoholic fatty liver disease (NAFLD) patients including those with NASH has been increasing worldwide and a portion of NASH patients will progress to hepatocarcinoma onset [121-123]. Therefore, numerous investigations have been performed in efforts to elucidate the causes of NASH.

$\mathrm{NASH}$ is characterized by fat deposition, inflammation and fibrosis in the liver, and a two-hit mechanism of onset has been proposed [124-126]. This hypothesis is that fatty liver formation and subsequent injuries, including inflammation and oxidative stress, cause NASH pathology [127]. Interestingly, recent studies have raised the possibility that UA is among the risk factors for NASH pathology. We discuss the relationship between UA and NASH below.

\subsubsection{Serum UA Is a Predictor of NAFLD/NASH Onset and}

Progression. Many clinical studies have been carried out to investigate the relationship between serum UA levels and NAFLD/NASH progression. For example, a cohort study in Korea found the serum UA level to be a useful marker for predicting NAFLD development because the serum UA concentration correlated positively with the 5-year incidence rate of NAFLD [128]. Their conclusion is supported by another study showing that serum UA levels of NAFLD patients are higher than those of control groups [129]. In addition, there are also studies demonstrating that serum UA is a risk factor for the development and/or progression of NAFLD including NASH [130-132].

Consistent with these observations, hepatic $\mathrm{XO}$ activities and serum UA levels are reportedly increased in murine NAFLD/NASH models $[133,134]$. Moreover, a fraction of NAFLD/NASH patients also have obesity, and hypertrophic adipocytes were also reported to secrete UA [135]. Taken together, these results indicate serum UA to be a good parameter for predicting the development of NAFLD/NASH, and that $\mathrm{XO}$ inhibitors or uricosuric agents might have potential as treatments for ameliorating the features of NAFLD.

\subsubsection{The Mechanism of UA-Induced NAFLD/NASH Pro-} gression. As described above, increasing serum UA or XO activity apparently plays important roles in NAFLD/NASH onset and progression. Interestingly, UA was reported to induce fat depositions by enhancing lipogenesis in hepatocytes. Fructose treatment of HepG 2 cells reportedly increased both the intracellular UA concentration and triglyceride (TG) accumulation, while allopurinol, an XO inhibitor, suppressed this fructose-mediated TG deposition. Moreover, the application of UA alone was demonstrated to increase intracellular TG contents as well as ROS generation in mitochondria [136]. As a mechanism of UA-induced TG accumulation, the authors asserted that the elevation of intracellular ROS by UA raised both the citrate concentration and ATP citrate lyase activity via enhanced phosphorylation at S455, resulting in the induction of lipogenesis. These observations are supported by those of another study in which pretreatment with antioxidants inhibited the elevation of triglyceride contents by UA [137]. The authors asserted that ROS generation by UA evoked endoplasmic reticulum stress, leading to upregulation of lipogenic genes, such as acetyl CoA carboxylasel and FASN [137].

ROS generation by UA is considered to depend on NADPH oxidase activation [136, 138, 139]. For example, UA reportedly promotes translocation of the NADPH oxidase subunit NOX4 into mitochondria [136]. It was also reported that UA treatment raises NADPH oxidase activity and alters its localization, leading to lipid oxidation [139]. In addition, $\mathrm{XO}$ may also function as a source of ROS generation because $\mathrm{XO}$ activity is upregulated in the livers of murine $\mathrm{NASH}$ models.

Collectively, these observations indicate that UA enhances fatty acid synthesis by regulating lipogenesis and induces ROS generation by regulating NADPH oxidase activity and upregulating fatty acid synthesis, thereby contributing to NASH development.

\subsubsection{Inflammasome Participation in NASH Progression.} As described elsewhere, UA is involved in inflammasome activation. Recent investigations have provided convincing evidence that inflammasomes are key players in NASH development. An initial study revealed that inflammasome impairment exacerbated the NASH progression induced by feeding a methionine-choline deficient diet for 4 weeks to ASC or IL-1 KO mice [140]. Subsequent studies, however, found that inflammasomes themselves exacerbate NASH symptoms. For example, it was reported that NLRP3 deficiency prevents liver fibrosis in response to a choline diet deficient in amino acids [141]. In addition, caspase-1 deficient mice were also resistant to developing steatosis or fibrosis while being fed a high-fat diet [142]. Moreover, other groups have demonstrated that diets which lead to NASH also increase the expressions of inflammasome components [143-145].

Taking these lines of evidence together, in the initial stage of NASH, inflammasomes appear to exert a protective effect, but continuous inflammasome activation appears to cause excessive productions of inflammatory cytokines, ultimately resulting in liver injury. Although, to date, numerous factors playing important roles in NASH progression have been identified, UA also appears to be a key participant in the onset of NAFLD/NASH.

3.3. Insulin Resistance, Diabetes, and Hyperlipidemia. Hyperuricemia was reportedly found to be related to insulin resistance in several clinical analyses [146-152]. In addition, several meta-analyses have suggested the UA level to be positively associated with the development of type 2 diabetes mellitus (DM) [153-156], although Mendelian randomization studies did not support circulating UA as being among the causes of DM development $[157,158]$. In metabolic syndrome patients, an oxidative stress marker, the myeloperoxidase level, was decreased by allopurinol and endothelial function improved [159]. On the other hand, rapid UA reduction 


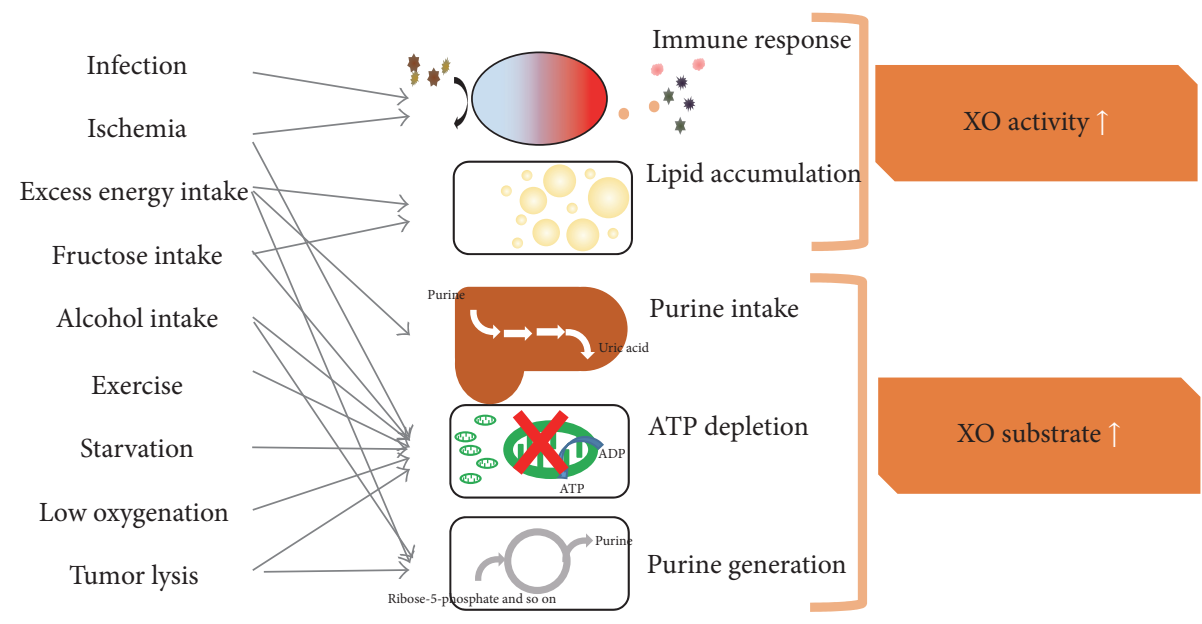

FIGURE 4: Increased catalyst activity of XO, originating from pathological and physiological events. Involvement of XO in pathophysiological processes suggests applications of XO inhibitors to the treatment of various disorders.

achieved by rasburicase, a urate oxidase, in obese subjects with high UA resulted in increasing the markers of systemic and skeletal muscle oxidative stress while having no effect on insulin sensitivity [160].

Furthermore, excess fructose intake is one of the major causes of the development of obesity with hyperuricemia, fatty liver, and metabolic syndrome. Fructose is metabolized by fructokinase to fructose-1-phosphate and results in a drop in both intracellular phosphate and ATP levels [161]. The intracellular phosphate decrease stimulates AMP deaminase (AMPD), the enzyme catalyzing the degradation of AMP to inosine monophosphate and eventually UA. Activated AMPD increases the expressions of gluconeogenesis genes, that is, PEPCK and G6Pase, via inhibition of AMP-activated protein kinase (AMPK) [162]. AMPD also increases lipogenesis through AMPK inhibition. AMPK phosphorylation was decreased in HepG2 cells treated with UA. The UA increased fructose-induced TG accumulation and decreased $\beta$-hydroxybutyrate levels, dose-dependently, while allopurinol, a XO inhibitor, blocked it. Because UA is the downstream product of AMPD and allopurinol abolished fructose-induced lipid accumulation, AMPD effects on AMPK appeared to depend on UA [163]. UA activates the transcription factor ChREBP, which triggers a vicious cycle of fructokinase transcription and accelerated fructose metabolism [68]. Via these mechanisms, activated AMPD and increased UA production tend to promote fat accumulation and glucose production.

UA is considered to be an antioxidant in human blood, though UA induces oxidative stress in cells [164]. UA raised NADPH oxidase activity and ROS production in mature adipocytes. The stimulation of NADPH oxidase-dependent ROS by UA resulted in the activation of MAP kinase p38 and ERK1/2, a decrease in NO bioavailability, and increases in both protein nitrosylation and lipid oxidation [138]. Increased UA production, in turn, generates mitochondrial oxidants. Mitochondrial oxidative stress inhibits aconitase in the Krebs cycle, resulting in citrate accumulation and the stimulation of ATP citrate lyase and fatty acid synthase, ultimately leading to de novo lipogenesis [136]. In hepatocytes treated with high UA, oxidative stress is increased, which activates serine (rat Ser307 and human Ser312) phosphorylation of IRS-1. This activity impairs Akt phosphorylation, thereby resulting in acute hepatic insulin resistance after exposure to high UA levels [165]. Therefore, UA-induced lipid accumulation and oxidative stress are responsible for the development of insulin resistance and diabetes.

\section{Beneficial Effects of XO Inhibitors}

Involvement of increased $\mathrm{XO}$ catalyst activity in pathophysiological processes (Figure 4) suggests applications of $\mathrm{XO}$ inhibitors to the treatment of various disorders. At present, XO inhibitors, including allopurinol, oxypurinol, febuxostat, and topiroxostat, are widely used for treating gout and hyperuricemia. Furthermore, XO inhibitors have been experimentally or clinically shown to exert beneficial effects by lowering serum UA and oxidative stress.

Febuxostat preserved renal function in 5/6 nephrectomized rats with and without coexisting hyperuricemia and prevented diabetic renal injury in streptozotocin-treated rats [166, 167]. Febuxostat also ameliorated tubular damage, diminished macrophage interstitial infiltration, and suppressed both proinflammatory cytokine activities and oxidative stress [168]. Febuxostat also reduced the induction of endoplasmic reticulum stress, as assessed by GRP-78 (glucose-regulated protein-78), ATF4 (activating transcription factor-4), and $\mathrm{CHOP}$ (C/EBP homologous protein-10) [169]. The clinical significance of measuring the serum UA level and XO inhibition for renal protection has largely been established by the results of recent studies [170-173].

On the other hand, beneficial effects of XO inhibitors on atherosclerosis and NASH constitute an evolving concept that has yet to be proven. In rats with fructose-induced metabolic syndrome, febuxostat treatment reversed hyperuricemia, hypertension, dyslipidemia, and insulin resistance [174]. The beneficial effects of XO inhibitors on NASH are 
rarely reported, except by our research group [134], because animal models of NASH with obesity, inflammation, and fibrosis have been difficult to establish. NASH in response to the MCD diet, as used in our studies, caused primarily inflammation and also made the mice lean, such that no benefit of XO inhibition was obtained [134]. Thus, we next used a high-fat diet containing trans-fatty acids and a highfructose diet to induce NASH development in our animal models. Another report showed that inhibition of XO activity also significantly prevents hepatic steatosis induced by a highfat diet in mice. XO has also been indicated to regulate activation of the NLRP3 inflammasome [175].

Atherosclerosis has been far more extensively investigated than NASH, both clinically and experimentally. Tungsten, acting as an XO inhibitor, has an inhibitory effect on both atherosclerosis and oxidative stress [176]. We reported for the first time that more specific XO inhibition, using allopurinol rather than tungsten on macrophages, resulted in the inhibition of foam cell formation and reduced atherosclerotic lesions in ApoE-KO mice, independently of the serum lipid profile [75]. We also identified phenotypic changes of macrophages in response to allopurinol, such as alterations of gene expressions involved in lipid accumulation. Moreover, both $\mathrm{XO}$ overexpression and knockdown of XO expression revealed VLDL receptors to be dramatically upregulated by XO. Febuxostat was also proven to have similar effects in terms of reducing the atherosclerotic lesions in ApoE$\mathrm{KO}$ mice, and oxidative stress was reduced in macrophages from atherosclerotic lesions [113]. Febuxostat also suppressed LPS-induced MCP-1 production via MAPK phosphatase-1mediated inactivation of JNK [84]. As a strategy for suppressing atherosclerosis, $\mathrm{XO}$ inhibition is expected to act on either macrophages or inflammatory cells.

$\mathrm{XO}$ inhibitors also improve endothelial function and prevent vascular remodeling. Oxypurinol reduces $\mathrm{O}_{2}{ }^{-}$radical dot production and improves endothelial function in blood vessels from hyperlipidemic experimental animals [69]. $\mathrm{XO}$ inhibition can also provide protection from radiationinduced endothelial dysfunction and cardiovascular complications [177]. Allopurinol treatment prevents hypoxiainduced vascular remodeling in the lung [178]. However, controversy persists as to whether the effect of $\mathrm{XO}$ on endothelial function is clinically relevant as an interventional target [49]. Pretreatment with XO inhibitors has beneficial effects on ischemia/reperfusion injuries of the intestine [179], in the impaired liver [61, 62], the edematous brain [180], kidneys with contrast induced nephropathy [181], and coronary ischemia [182]. XO inhibitors prevent postischemic $\mathrm{O}_{2}{ }^{-}$ generation [183].

\section{Conclusion}

Inflammation related to UA metabolism is induced via either inflammasome activation by UA crystal precipitation or free radical production in response to $\mathrm{XO}$ activity. In addition to gout, many disorders are known to be related to UA metabolism and XO inhibitor treatments have been shown to be effective for preventing the onset and/or the progression of such disorders. In particular, atherosclerosis and NASH are diseases for which relationships with UA metabolism were not immediately recognized, but rodent model studies revealed the importance of UA metabolism maintenance for managing these disorders. We believe the impact of UA metabolism on many diseases accompanying chronic inflammation to have been underestimated. Future studies are anticipated to reveal the pathological contribution of serum UA and/or XO activity to the specific processes underlying various disorders. Further study of the detailed molecular mechanisms is clearly warranted.

\section{Abbreviations \\ UA: $\quad$ Uric acid \\ MUC: Monosodium urate crystal \\ NASH: Nonalcoholic steatohepatitis \\ XO: Xanthine oxidase \\ LPS: Lipopolysaccharide \\ TIMP: Tissue inhibitor of metalloproteinases \\ MCP-1: Monocyte chemoattractant protein 1 \\ NADPH: Nicotinamide adenine dinucleotide phosphate \\ CKD: Chronic kidney disease \\ ICAM-1: Intercellular adhesion molecule-1.}

\section{Competing Interests}

The authors have no competing interests regarding the publication of this report to declare.

\section{References}

[1] C. E. Berry and J. M. Hare, "Xanthine oxidoreductase and cardiovascular disease: molecular mechanisms and pathophysiological implications," Journal of Physiology, vol. 555, no. 3, pp. 589-606, 2004.

[2] B. N. Ames, R. Cathcart, E. Schwiers, and P. Hochstein, "Uric acid provides an antioxidant defense in humans against oxidant- and radical-caused aging and cancer: a hypothesis," Proceedings of the National Academy of Sciences of the United States of America, vol. 78, no. 11, pp. 6858-6862, 1981.

[3] C. X. C. Santos, E. I. Anjos, and O. Augusto, "Uric acid oxidation by peroxynitrite: multiple reactions, free radical formation, and amplification of lipid oxidation," Archives of Biochemistry and Biophysics, vol. 372, no. 2, pp. 285-294, 1999.

[4] E. Kamogawa and Y. Sueishi, "A multiple free-radical scavenging (MULTIS) study on the antioxidant capacity of a neuroprotective drug, edaravone as compared with uric acid, glutathione, and trolox," Bioorganic \& Medicinal Chemistry Letters, vol. 24, no. 5, pp. 1376-1379, 2014.

[5] C. Gersch, S. P. Palii, K. M. Kim, A. Angerhofer, R. J. Johnson, and G. N. Henderson, "Inactivation of nitric oxide by uric acid," Nucleosides, Nucleotides and Nucleic Acids, vol. 27, no. 8, pp. 967-978, 2008.

[6] R. A. Patterson, E. T. M. Horsley, and D. S. Leake, "Prooxidant and antioxidant properties of human serum ultrafiltrates toward LDL: important role of uric acid," Journal of Lipid Research, vol. 44, no. 3, pp. 512-521, 2003.

[7] J. R. León-Carmona and A. Galano, "Uric and 1-methyluric acids: metabolic wastes or antiradical protectors?" Journal of Physical Chemistry B, vol. 115, no. 51, pp. 15430-15438, 2011. 
[8] J. W. Davis, A. Grandinetti, C. I. Waslien, G. W. Ross, L. R. White, and D. M. Morens, "Observations on serum uric acid levels and the risk of idiopathic Parkinson's disease," American Journal of Epidemiology, vol. 144, no. 5, pp. 480-484, 1996.

[9] J. Drulović, I. Dujmović, N. Stojsavljević et al., "Uric acid levels in sera from patients with multiple sclerosis," Journal of Neurology, vol. 248, no. 2, pp. 121-126, 2001.

[10] G. Toncev, B. Milicic, S. Toncev, and G. Samardzic, "Serum uric acid levels in multiple sclerosis patients correlate with activity of disease and blood-brain barrier dysfunction," European Journal of Neurology, vol. 9, no. 3, pp. 221-226, 2002.

[11] B. Liu, Y. Shen, K. Xiao, Y. Tang, L. Cen, and J. Wei, "Serum uric acid levels in patients with multiple sclerosis: a meta-analysis," Neurological Research, vol. 34, no. 2, pp. 163-171, 2012.

[12] F. Ashtari, M. Bahar, M. Aghaei, and A. Zahed, "Serum uric acid level in patients with relapsing-remitting multiple sclerosis," Journal of Clinical Neuroscience, vol. 20, no. 5, pp. 676-678, 2013.

[13] D. Keizman, M. Ish-Shalom, S. Berliner et al., "Low uric acid levels in serum of patients with ALS: further evidence for oxidative stress?" Journal of the Neurological Sciences, vol. 285, no. 1-2, pp. 95-99, 2009.

[14] S. Paganoni, M. Zhang, A. Q. Zárate et al., "Uric acid levels predict survival in men with amyotrophic lateral sclerosis," Journal of Neurology, vol. 259, no. 9, pp. 1923-1928, 2012.

[15] F. Martinon, K. Burns, and J. Tschopp, “The Inflammasome: a molecular platform triggering activation of inflammatory caspases and processing of proIL- $\beta$," Molecular Cell, vol. 10, no. 2, pp. 417-426, 2002.

[16] K. Schroder and J. Tschopp, “The inflammasomes," Cell, vol. 140, no. 6, pp. 821-832, 2010.

[17] R. Medzhitov, "Origin and physiological roles of inflammation," Nature, vol. 454, no. 7203, pp. 428-435, 2008.

[18] F. Martinon, V. Pétrilli, A. Mayor, A. Tardivel, and J. Tschopp, "Gout-associated uric acid crystals activate the NALP3 inflammasome," Nature, vol. 440, no. 7081, pp. 237-241, 2006.

[19] K. L. Rock, H. Kataoka, and J.-J. Lai, "Uric acid as a danger signal in gout and its comorbidities," Nature Reviews Rheumatology, vol. 9, no. 1, pp. 13-23, 2013.

[20] K. H. G. Mills, L. S. Dungan, S. A. Jones, and J. Harris, "The role of inflammasome-derived IL-1 in driving IL-17 responses," Journal of Leukocyte Biology, vol. 93, no. 4, pp. 489-497, 2013.

[21] P. P. Duewell, H. Kono, K. J. Rayner et al., "NLRP3 inflammasomes are required for atherogenesis and activated by cholesterol crystals," Nature, vol. 464, no. 7293, pp. 1357-1361, 2010.

[22] B. Razani, C. Feng, T. Coleman et al., "Autophagy links inflammasomes to atherosclerotic progression," Cell Metabolism, vol. 15, no. 4, pp. 534-544, 2012.

[23] T. Csak, M. Ganz, J. Pespisa, K. Kodys, A. Dolganiuc, and G. Szabo, "Fatty acid and endotoxin activate inflammasomes in mouse hepatocytes that release danger signals to stimulate immune cells," Hepatology, vol. 54, no. 1, pp. 133-144, 2011.

[24] G. C. Farrell, D. Van Rooyen, L. Gan, and S. Chitturi, "NASH is an inflammatory disorder: pathogenic, prognostic and therapeutic implications," Gut and Liver, vol. 6, no. 2, pp. 149-171, 2012.

[25] P. Gasse, N. Riteau, S. Charron et al., "Uric acid is a danger signal activating NALP3 inflammasome in lung injury inflammation and fibrosis," American Journal of Respiratory and Critical Care Medicine, vol. 179, no. 10, pp. 903-913, 2009.

[26] V. Pétrilli, S. Papin, C. Dostert, A. Mayor, F. Martinon, and J. Tschopp, "Activation of the NALP3 inflammasome is triggered by low intracellular potassium concentration," Cell Death and Differentiation, vol. 14, no. 9, pp. 1583-1589, 2007.

[27] J.-J. Jhang, Y.-T. Cheng, C.-Y. Ho, and G.-C. Yen, "Monosodium urate crystals trigger Nrf2- and heme oxygenase-1-dependent inflammation in THP-1 cells," Cellular \& Molecular Immunology, vol. 12, no. 4, pp. 424-434, 2015.

[28] J. Nomura, A. So, M. Tamura, and N. Busso, "Intracellular ATP decrease mediates NLRP3 inflammasome activation upon nigericin and crystal stimulation," The Journal of Immunology, vol. 195, no. 12, pp. 5718-5724, 2015.

[29] R. Zhou, A. Tardivel, B. Thorens, I. Choi, and J. Tschopp, "Thioredoxin-interacting protein links oxidative stress to inflammasome activation," Nature Immunology, vol. 11, no. 2, pp. 136-140, 2010.

[30] K. Schroder, R. Zhou, and J. Tschopp, "The NLRP3 inflammasome: a sensor for metabolic danger?” Science, vol. 327, no. 5963, pp. 296-300, 2010.

[31] A. Mittal, A. R. J. Phillips, B. Loveday, and J. A. Windsor, "The potential role for xanthine oxidase inhibition in major intraabdominal surgery," World Journal of Surgery, vol. 32, no. 2, pp. 288-295, 2008.

[32] R. Asai, T. Nishino, T. Matsumura et al., "Two mutations convert mammalian xanthine oxidoreductase to highly superoxideproductive xanthine oxidase," Journal of Biochemistry, vol. 141, no. 4, pp. 525-534, 2007.

[33] A. Papi, M. Contoli, P. Gasparini et al., "Role of xanthine oxidase activation and reduced glutathione depletion in rhinovirus induction of inflammation in respiratory epithelial cells," Journal of Biological Chemistry, vol. 283, no. 42, pp. 28595-28606, 2008.

[34] M. J. Barber, R. C. Bray, R. Cammack, and M. P. Coughlan, "Oxidation-reduction potentials of turkey liver xanthine dehydrogenase and the origins of oxidase and dehydrogenase behaviour in molybdenum-containing hydroxylases," Biochemical Journal, vol. 163, no. 2, pp. 279-289, 1977.

[35] H. Li, S. Horke, and U. Förstermann, "Oxidative stress in vascular disease and its pharmacological prevention," Trends in Pharmacological Sciences, vol. 34, no. 6, pp. 313-319, 2013.

[36] E. A. Herrera, A. D. Kane, J. A. Hansell et al., "A role for xanthine oxidase in the control of fetal cardiovascular function in late gestation sheep," Journal of Physiology, vol. 590, no. 8, pp. 18251837, 2012.

[37] A. D. Kane, J. A. Hansell, E. A. Herrera et al., "Xanthine oxidase and the fetal cardiovascular defence to hypoxia in late gestation ovine pregnancy," Journal of Physiology, vol. 592, no. 3, pp. 475489, 2014.

[38] T. Ohtsubo, K. Matsumura, K. Sakagami et al., "Xanthine oxidoreductase depletion induces renal interstitial fibrosis through aberrant lipid and purine accumulation in renal tubules," Hypertension, vol. 54, no. 4, pp. 868-876, 2009.

[39] C. A. Pritsos, "Cellular distribution, metabolism and regulation of the xanthine oxidoreductase enzyme system," ChemicoBiological Interactions, vol. 129, no. 1-2, pp. 195-208, 2000.

[40] H. M. Martin, K. P. Moore, E. Bosmans et al., "Xanthine oxidoreductase is present in bile ducts of normal and cirrhotic liver," Free Radical Biology and Medicine, vol. 37, no. 8, pp. 12141223, 2004.

[41] X. Wang, D. Oberleas, M. T. Yang, and S. P. Yang, "Molybdenum requirement of female rats," Journal of Nutrition, vol. 122, no. 4, pp. 1036-1041, 1992. 
[42] Y. Moriwaki, T. Yamamoto, M. Suda et al., "Purification and immunohistochemical tissue localization of human xanthine oxidase," Biochimica et Biophysica Acta (BBA)_Protein Structure and Molecular Enzymology, vol. 1164, no. 3, pp. 327-330, 1993.

[43] J. W. De Jong, R. G. Schoemaker, R. De Jonge et al., "Enhanced expression and activity of xanthine oxidoreductase in the failing heart," Journal of Molecular and Cellular Cardiology, vol. 32, no. 11, pp. 2083-2089, 2000.

[44] Y. Ohara, T. E. Peterson, and D. G. Harrison, "Hypercholesterolemia increases endothelial superoxide anion production," Journal of Clinical Investigation, vol. 91, no. 6, pp. 2546-2551, 1993.

[45] S. Spiekermann, U. Landmesser, S. Dikalov et al., "Electron spin resonance characterization of vascular xanthine and $\mathrm{NAD}(\mathrm{P}) \mathrm{H}$ oxidase activity in patients with coronary artery disease: relation to endothelium-dependent vasodilation," Circulation, vol. 107, no. 10, pp. 1383-1389, 2003.

[46] C.-C. Yang, M.-C. Ma, C.-T. Chien, M.-S. Wu, W.-K. Sun, and C.-F. Chen, "Hypoxic preconditioning attenuates lipopolysaccharide-induced oxidative stress in rat kidneys," The Journal of Physiology, vol. 582, no. 1, pp. 407-419, 2007.

[47] U. Landmesser, S. Spiekermann, C. Preuss et al., "Angiotensin II induces endothelial xanthine oxidase activation: role for endothelial dysfunction in patients with coronary disease," Arteriosclerosis, Thrombosis, and Vascular Biology, vol. 27, no. 4, pp. 943-948, 2007.

[48] S. A. Nicholas, V. V. Bubnov, I. M. Yasinska, and V. V. Sumbayev, "Involvement of xanthine oxidase and hypoxia-inducible factor 1 in Toll-like receptor 7/8-mediated activation of caspase 1 and interleukin-1 $\beta$," Cellular and Molecular Life Sciences, vol. 68, no. 1, pp. 151-158, 2011.

[49] U. Förstermann, "Nitric oxide and oxidative stress in vascular disease," Pflugers Archiv-European Journal of Physiology, vol. 459, no. 6, pp. 923-939, 2010.

[50] C. R. White, V. Darley-Usmar, W. R. Berrington et al., "Circulating plasma xanthine oxidase contributes to vascular dysfunction in hypercholesterolemic rabbits," Proceedings of the National Academy of Sciences of the United States of America, vol. 93, no. 16, pp. 8745-8749, 1996.

[51] O. Elmas, O. Elmas, and S. Caliskan, "Investigation of the oxidative effect of chronic hyperammonemia on the kidney and the possible protective effect of allopurinol," Renal Failure, vol. 33, no. 1, pp. 61-65, 2011.

[52] M. Faruk Hossain, M. Ismail, A. R. Tanu, and H. Uddin Shekhar, "Respiratory burst enzymes, pro-oxidants and antioxidants status in bangladeshi population with $\beta$-thalassemia major," North American Journal of Medical Sciences, vol. 7, no. 6, pp. 253-258, 2015.

[53] G. Chen, J. Yang, G. Lu, J. Guo, and Y. Dou, "Limb remote ischemic post-conditioning reduces brain reperfusion injury by reversing eNOS uncoupling," Indian Journal of Experimental Biology, vol. 52, no. 6, pp. 597-605, 2014.

[54] L. A. Gabrielli, P. F. Castro, I. Godoy et al., "Systemic oxidative stress and endothelial dysfunction is associated with an attenuated acute vascular response to inhaled prostanoid in pulmonary artery hypertension patients," Journal of Cardiac Failure, vol. 17, no. 12, pp. 1012-1017, 2011.

[55] R. Aranda, E. Doménech, A. D. Rus et al., "Age-related increase in xanthine oxidase activity in human plasma and rat tissues," Free Radical Research, vol. 41, no. 11, pp. 1195-1200, 2007.
[56] Y. Aliciguzel, I. Ozen, M. Aslan, and U. Karayalcin, "Activities of xanthine oxidoreductose and antioxidant enzymes in different tissues of diabetic rats," The Journal of Laboratory and Clinical Medicine, vol. 142, no. 3, pp. 172-177, 2003.

[57] L. Malardé, A. Rebillard, S. Le Douairon-Lahaye et al., "Superoxide production pathways in aortas of diabetic rats: beneficial effects of insulin therapy and endurance training," Molecular and Cellular Biochemistry, vol. 389, no. 1-2, pp. 113-118, 2014.

[58] D. A. Peterson, R. W. Asinger, K. J. Elsperger, D. C. Homans, and J. W. Eaton, "Reactive oxygen species may cause myocardial reperfusion injury," Biochemical and Biophysical Research Communications, vol. 127, no. 1, pp. 87-93, 1985.

[59] T. Ono, R. Tsuruta, M. Fujita et al., "Xanthine oxidase is one of the major sources of superoxide anion radicals in blood after reperfusion in rats with forebrain ischemia/reperfusion," Brain Research, vol. 1305, pp. 158-167, 2009.

[60] M. Portugal-Cohen and R. Kohen, "Exposure of human keratinocytes to ischemia, hyperglycemia and their combination induces oxidative stress via the enzymes inducible nitric oxide synthase and xanthine oxidase," Journal of Dermatological Science, vol. 55, no. 2, pp. 82-90, 2009.

[61] M. O. Taha, M. J. Simões, E. C. Noguerol et al., "Effects of allopurinol on ischemia and reperfusion in rabbit livers," Transplantation Proceedings, vol. 41, no. 3, pp. 820-823, 2009.

[62] S. Peglow, A. H. Toledo, R. Anaya-Prado, F. Lopez-Neblina, and L. H. Toledo-Pereyra, "Allopurinol and xanthine oxidase inhibition in liver ischemia reperfusion," Journal of HepatoBiliary-Pancreatic Sciences, vol. 18, no. 2, pp. 137-146, 2011.

[63] T. Yoshikawa, S. Ueda, Y. Naito et al., "Role of oxygen-derived free radicals in gastric mucosal injury induced by ischemia or ischemia-reperfusion in rats," Free Radical Research Communications, vol. 7, no. 3-7, pp. 285-291, 1989.

[64] V. L. Vega, L. Mardones, M. Maldonado et al., "Xanthine oxidase released from reperfused hind limbs mediate Küpffer cell activation, neutrophil sequestration, and hepatic oxidative stress in rats subjected to tourniquet shock," Shock, vol. 14, no. 5, pp. 565-571, 2000.

[65] J. D. Vaghasiya, N. R. Sheth, Y. S. Bhalodia, and N. P. Jivani, "Exaggerated liver injury induced by renal ischemia reperfusion in diabetes: effect of exenatide," Saudi Journal of Gastroenterology, vol. 16, no. 3, pp. 174-180, 2010.

[66] J. Hallfrisch, "Metabolic effects of dietary fructose," The FASEB Journal, vol. 4, no. 9, pp. 2652-2660, 1990.

[67] F. Stirpe, E. Della Corte, E. Bonetti, A. Abbondanza, A. Abbati, and F. De Stefano, "Fructose-induced hyperuricaemia," The Lancet, vol. 296, no. 7686, pp. 1310-1311, 1970.

[68] M. A. Lanaspa, L. G. Sanchez-Lozada, C. Cicerchi et al., "Uric acid stimulates fructokinase and accelerates fructose metabolism in the development of fatty liver," PLOS ONE, vol. 7, no. 10, Article ID e47948, 2012.

[69] T. F. Roe and M. D. Kogut, "The pathogenesis of hyperuricemia in glycogen storage disease, type I," Pediatric Research, vol. 11, no. 5, pp. 664-669, 1977.

[70] N. B. Medvedeva, P. L. Telushkin, and A. Y. Stel'makh, "Parameters of nitrogen metabolism during insulin hypoglycemia in rats with alloxan-induced diabetes," Bulletin of Experimental Biology and Medicine, vol. 146, no. 2, pp. 203-205, 2008.

[71] S. Sachdev and K. J. A. Davies, "Production, detection, and adaptive responses to free radicals in exercise," Free Radical Biology and Medicine, vol. 44, no. 2, pp. 215-223, 2008. 
[72] M. F. Vincent, G. Van den Berghe, and H. G. Hers, “The pathway of adenine nucleotide catabolism and its control in isolated rat hepatocytes subjected to anoxia," Biochemical Journal, vol. 202, no. 1, pp. 117-123, 1982.

[73] H. Biri, H. S. Öztürk, M. Kaçmaz, K. Karaca, H. Tokuçoǧlu, and I. Durak, "Activities of DNA turnover and free radical metabolizing enzymes in cancerous human prostate tissue," Cancer Investigation, vol. 17, no. 5, pp. 314-319, 1999.

[74] K. Kishimoto, R. Kobayashi, M. Ichikawa et al., "Risk factors for tumor lysis syndrome in childhood acute myeloid leukemia treated with a uniform protocol without rasburicase prophylaxis," Leukemia \& Lymphoma, vol. 56, no. 7, pp. 2193-2195, 2015.

[75] A. Kushiyama, H. Okubo, H. Sakoda et al., "Xanthine oxidoreductase is involved in macrophage foam cell formation and atherosclerosis development," Arteriosclerosis, Thrombosis, and Vascular Biology, vol. 32, no. 2, pp. 291-298, 2012.

[76] T. Ohtsubo, I. I. Rovira, M. F. Starost, C. Liu, and T. Finkel, "Xanthine oxidoreductase is an endogenous regulator of cyclooxygenase-2," Circulation Research, vol. 95, no. 11, pp. 11181124, 2004.

[77] C. Vorbach, R. Harrison, and M. R. Capecchi, "Xanthine oxidoreductase is central to the evolution and function of the innate immune system," Trends in Immunology, vol. 24, no. 9, pp. 512-517, 2003.

[78] T. Hartney, R. Birari, S. Venkataraman et al., "Xanthine oxidasederived ROS upregulate Egr-1 via ERK1/2 in PA smooth muscle cells; model to test impact of extracellular ROS in chronic hypoxia," PLoS ONE, vol. 6, no. 11, Article ID e27531, 2011.

[79] S. Gibbings, N. D. Elkins, H. Fitzgerald et al., "Xanthine oxidoreductase promotes the inflammatory state of mononuclear phagocytes through effects on chemokine expression, peroxisome proliferator-activated receptor- $\gamma$ sumoylation, and HIF-1 $\alpha$," Journal of Biological Chemistry, vol. 286, no. 2, pp. 961975, 2011.

[80] E. Morse, J. Schroth, Y.-H. You et al., "TRB3 is stimulated in diabetic kidneys, regulated by the ER stress marker CHOP, and is a suppressor of podocyte MCP-1," American Journal of Physiology-Renal Physiology, vol. 299, no. 5, pp. F965-F972, 2010.

[81] D. B. Corry, P. Eslami, K. Yamamoto, M. D. Nyby, H. Makino, and M. L. Tuck, "Uric acid stimulates vascular smooth muscle cell proliferation and oxidative stress via the vascular reninangiotensin system," Journal of Hypertension, vol. 26, no. 2, pp. 269-275, 2008.

[82] T. Kosugi, T. Nakayama, M. Heinig et al., "Effect of lowering uric acid on renal disease in the type 2 diabetic $\mathrm{db} / \mathrm{db}$ mice," American Journal of Physiology-Renal Physiology, vol. 297, no. 2, pp. F481-F488, 2009.

[83] E.-S. Ryu, M. J. Kim, H.-S. Shin et al., "Uric acid-induced phenotypic transition of renal tubular cells as a novel mechanism of chronic kidney disease," American Journal of Physiology-Renal Physiology, vol. 304, no. 5, pp. F471-F480, 2013.

[84] J. Nomura, N. Busso, A. Ives et al., "Febuxostat, an inhibitor of xanthine oxidase, suppresses lipopolysaccharide-induced MCP-1 production via MAPK phosphatase-1-mediated inactivation of JNK," PLOS ONE, vol. 8, no. 9, Article ID e75527, 2013.

[85] N. Davis, "The cardiovascular and renal relations and manifestations of gout," JAMA, vol. 29, pp. 261-262, 1897.

[86] D. I. Feig, D.-H. Kang, and R. J. Johnson, "Uric acid and cardiovascular risk," The New England Journal of Medicine, vol. 359, no. 17, pp. 1811-1821, 2008.
[87] J. V. Selby, G. D. Friedman, and C. P. Quesenberry Jr, "Precursors of essential hypertension: pulmonary function, heart rate, uric acid, serum cholesterol, and other serum chemistries," American Journal of Epidemiology, vol. 131, no. 6, pp. 1017-1027, 1990.

[88] S. C. Hunt, S. H. Stephenson, P. N. Hopkins, and R. R. Williams, "Predictors of an increased risk of future hypertension in Utah. A screening analysis," Hypertension, vol. 17, no. 6, pp. 969-976, 1991.

[89] D. I. Feig and R. J. Johnson, "Hyperuricemia in childhood primary hypertension," Hypertension, vol. 42, no. 3, pp. 247-252, 2003.

[90] J. Sundström, L. Sullivan, R. B. D’Agostino, D. Levy, W. B. Kannel, and R. S. Vasan, "Relations of serum uric acid to longitudinal blood pressure tracking and hypertension incidence," Hypertension, vol. 45, no. 1, pp. 28-33, 2005.

[91] T. S. Perlstein, O. Gumieniak, G. H. Williams et al., "Uric acid and the development of hypertension: The Normative Aging Study," Hypertension, vol. 48, no. 6, pp. 1031-1036, 2006.

[92] E. Krishnan, C. K. Kwoh, H. R. Schumacher, and L. Kuller, "Hyperuricemia and incidence of hypertension among men without metabolic syndrome," Hypertension, vol. 49, no. 2, pp. 298-303, 2007.

[93] J. P. Forman, H. Choi, and G. C. Curhan, "Uric acid and insulin sensitivity and risk of incident hypertension," Archives of Internal Medicine, vol. 169, no. 2, pp. 155-162, 2009.

[94] J. Wang, T. Qin, J. Chen et al., "Hyperuricemia and risk of incident hypertension: a systematic review and meta-analysis of observational studies," PLoS ONE, vol. 9, no. 12, Article ID el14259, 2014.

[95] M. Volterrani, F. Iellamo, B. Sposato, and F. Romeo, "Uric acid lowering therapy in cardiovascular diseases," International Journal of Cardiology, vol. 213, pp. 20-22, 2016.

[96] S. E. Perez-Pozo, J. Schold, T. Nakagawa, L. G. Sánchez-Lozada, R. J. Johnson, and J. L. Lillo, "Excessive fructose intake induces the features of metabolic syndrome in healthy adult men: role of uric acid in the hypertensive response," International Journal of Obesity, vol. 34, no. 3, pp. 454-461, 2010.

[97] D. I. Feig, B. Soletsky, and R. J. Johnson, "Effect of allopurinol on blood pressure of adolescents with newly diagnosed essential hypertension: a randomized trial," The Journal of the American Medical Association, vol. 300, no. 8, pp. 924-932, 2008.

[98] B. Soletsky and D. I. Feig, "Uric acid reduction rectifies prehypertension in obese adolescents," Hypertension, vol. 60, no. 5, pp. 1148-1156, 2012.

[99] V. Agarwal, N. Hans, and F. H. Messerli, "Effect of allopurinol on blood pressure: a systematic review and meta-analysis," Journal of Clinical Hypertension, vol. 15, no. 6, pp. 435-442, 2013.

[100] M. Mazzali, J. Hughes, Y.-G. Kim et al., "Elevated uric acid increases blood pressure in the rat by a novel crystalindependent mechanism," Hypertension, vol. 38, no. 5, pp. 11011106, 2001.

[101] M. Mazzali, J. Kanellis, L. Han et al., "Hyperuricemia induces a primary renal arteriolopathy in rats by a blood pressureindependent mechanism," American Journal of PhysiologyRenal Physiology, vol. 282, no. 6, pp. F991-F997, 2002.

[102] M. H. Alderman, H. Cohen, S. Madhavan, and S. Kivlighn, "Serum uric acid and cardiovascular events in successfully treated hypertensive patients," Hypertension, vol. 34, no. 1, pp. 144-150, 1999. 
[103] P. Verdecchia, G. Schillaci, G. Reboldi, F. Santeusanio, C. Porcellati, and P. Brunetti, "Relation between serum uric acid and risk of cardiovascular disease in essential hypertension: The PIUMA study," Hypertension, vol. 36, no. 6, pp. 1072-1078, 2000.

[104] M. J. Bos, P. J. Koudstaal, A. Hofman, J. C. M. Witteman, and M. M. B. Breteler, "Uric acid is a risk factor for myocardial infarction and stroke: the Rotterdam Study," Stroke, vol. 37, no. 6, pp. 1503-1507, 2006.

[105] H. K. Choi and G. Curhan, "Independent impact of gout on mortality and risk for coronary heart disease," Circulation, vol. 116, no. 8, pp. 894-900, 2007.

[106] A. G. Stack, A. Hanley, L. F. Casserly et al., "Independent and conjoint associations of gout and hyperuricaemia with total and cardiovascular mortality," QJM, vol. 106, no. 7, pp. 647-658, 2013.

[107] M. Li, X. Hu, Y. Fan et al., "Hyperuricemia and the risk for coronary heart disease morbidity and mortality a systematic review and dose-response meta-analysis," Scientific Reports, vol. 6, article 19520, 2016.

[108] L. Grimaldi-Bensouda, A. Alpérovitch, E. Aubrun et al., "Impact of allopurinol on risk of myocardial infarction," Annals of the Rheumatic Diseases, vol. 74, no. 5, pp. 836-842, 2015.

[109] A. Noman, D. S. Ang, S. Ogston, C. C. Lang, and A. D. Struthers, "Effect of high-dose allopurinol on exercise in patients with chronic stable angina: a randomised, placebo controlled crossover trial," The Lancet, vol. 375, no. 9732, pp. 2161-2167, 2010.

[110] J. George, E. Carr, J. Davies, J. J. F. Belch, and A. Struthers, "High-dose allopurinol improves endothelial function by profoundly reducing vascular oxidative stress and not by lowering uric acid," Circulation, vol. 114, no. 23, pp. 2508-2516, 2006.

[111] N. S. Rajendra, S. Ireland, J. George, J. J. F. Belch, C. C. Lang, and A. D. Struthers, "Mechanistic insights into the therapeutic use of high-dose allopurinol in angina pectoris," Journal of the American College of Cardiology, vol. 58, no. 8, pp. 820-828, 2011.

[112] K. Ogino, M. Kato, Y. Furuse et al., "Uric acid-lowering treatment with benzbromarone in patients with heart failure: a double-blind placebo-controlled crossover preliminary study," Circulation: Heart Failure, vol. 3, no. 1, pp. 73-81, 2010.

[113] Y. Moriwaki, T. Yamamoto, M. Suda et al., "Purification and immunohistochemical tissue localization of human xanthine oxidase," Biochimica et Biophysica Acta, vol. 1164, no. 3, pp. 327330, 1993.

[114] J. Nomura, N. Busso, A. Ives et al., "Xanthine oxidase inhibition by febuxostat attenuates experimental atherosclerosis in mice," Scientific Reports, vol. 4, article no. 4554, 2014.

[115] H. Cai and D. G. Harrison, "Endothelial dysfunction in cardiovascular diseases: the role of oxidant stress," Circulation Research, vol. 87, no. 10, pp. 840-844, 2000.

[116] K. J. Moore, F. J. Sheedy, and E. A. Fisher, "Macrophages in atherosclerosis: a dynamic balance," Nature Reviews Immunology, vol. 13, no. 10, pp. 709-721, 2013.

[117] P. Duewell, H. Kono, K. J. Rayner et al., "NLRP3 inflammasomes are required for atherogenesis and activated by cholesterol crystals," Nature, vol. 464, no. 7293, pp. 1357-1361, 2010.

[118] M. Mishima, T. Hamada, N. Maharani et al., "Effects of Uric Acid on the NO Production of HUVECs and its Restoration by Urate Lowering Agents," Drug Research, vol. 66, no. 05, pp. 270274, 2016.

[119] U. M. Khosla, S. Zharikov, J. L. Finch et al., "Hyperuricemia induces endothelial dysfunction," Kidney International, vol. 67, no. 5, pp. 1739-1742, 2005.
[120] G. N. Rao, M. A. Corson, and B. C. Berk, "Uric acid stimulates vascular smooth muscle cell proliferation by increasing plateletderived growth factor A-chain expression," The Journal of Biological Chemistry, vol. 266, no. 13, pp. 8604-8608, 1991.

[121] R. Loomba and A. J. Sanyal, "The global NAFLD epidemic," Nature Reviews Gastroenterology and Hepatology, vol. 10, no. 11, pp. 686-690, 2013.

[122] S. Mittal and H. B. El-Serag, "Epidemiology of hepatocellular carcinoma: consider the population," Journal of Clinical Gastroenterology, vol. 47, no. 1, pp. S2-S6, 2013.

[123] G. C. Farrell and C. Z. Larter, "Nonalcoholic fatty liver disease: from steatosis to cirrhosis," Hepatology, vol. 43, no. 2, pp. S99S112, 2006.

[124] D. E. Kleiner, E. M. Brunt, M. Van Natta et al., "Design and validation of a histological scoring system for nonalcoholic fatty liver disease. Nonalcoholic Steatohepatitis Clinical Research Network," Hepatology, vol. 41, no. 6, pp. 1313-1321, 2005.

[125] C. A. Matteoni, Z. M. Younossi, T. Gramlich, N. Boparai, Yao Chang Liu, and A. J. McCullough, "Nonalcoholic fatty liver disease: a spectrum of clinical and pathological severity," Gastroenterology, vol. 116, no. 6, pp. 1413-1419, 1999.

[126] J. D. Browning and J. D. Horton, "Molecular mediators of hepatic steatosis and liver injury," Journal of Clinical Investigation, vol. 114, no. 2, pp. 147-152, 2004.

[127] J. S. Lim, M. Mietus-Snyder, A. Valente, J.-M. Schwarz, and R. H. Lustig, "The role of fructose in the pathogenesis of NAFLD and the metabolic syndrome," Nature Reviews Gastroenterology and Hepatology, vol. 7, no. 5, pp. 251-264, 2010.

[128] J. W. Lee, Y. K. Cho, M. C. Ryan et al., "Serum uric acid as a predictor for the development of nonalcoholic fatty liver disease in apparently healthy subjects: A 5-Year Retrospective Cohort Study," Gut and Liver, vol. 4, no. 3, pp. 378-383, 2010.

[129] Y. Li, C. Xu, C. Yu, L. Xu, and M. Miao, "Association of serum uric acid level with non-alcoholic fatty liver disease: a crosssectional study," Journal of Hepatology, vol. 50, no. 5, pp. 10291034, 2009.

[130] S. Petta, C. Cammà, D. Cabibi, V. Di Marco, and A. Craxì, "Hyperuricemia is associated with histological liver damage in patients with non-alcoholic fatty liver disease," Alimentary Pharmacology \& Therapeutics, vol. 34, no. 7, pp. 757-766, 2011.

[131] E. Sertoglu, C. N. Ercin, G. Celebi et al., "The relationship of serum uric acid with non-alcoholic fatty liver disease," Clinical Biochemistry, vol. 47, no. 6, pp. 383-388, 2014.

[132] S. M. Lerret, L. Garcia-Rodriguez, J. Skelton, V. Biank, D. Kilway, and G. Telega, "Predictors of nonalcoholic steatohepatitis in obese children," Gastroenterology Nursing, vol. 34, no. 6, pp. 434-437, 2011.

[133] C. Xu, X. Wan, L. Xu et al., "Xanthine oxidase in non-alcoholic fatty liver disease and hyperuricemia: one stone hits two birds," Journal of Hepatology, vol. 62, no. 6, article 5522, pp. 1412-1419, 2015.

[134] Y. Nakatsu, Y. Seno, A. Kushiyama et al., "The xanthine oxidase inhibitor febuxostat suppresses development of nonalcoholic steatohepatitis in a rodent model," American Journal of Physiology-Gastrointestinal and Liver Physiology, vol. 309, no. 1, pp. G42-G51, 2015.

[135] Y. Tsushima, H. Nishizawa, Y. Tochino et al., "Uric acid secretion from adipose tissue and its increase in obesity," Journal of Biological Chemistry, vol. 288, no. 38, pp. 27138-27149, 2013.

[136] M. A. Lanaspa, L. G. Sanchez-Lozada, Y.-J. Choi et al., "Uric acid induces hepatic steatosis by generation of mitochondrial 
oxidative stress: potential role in fructose-dependent and independent fatty liver," Journal of Biological Chemistry, vol. 287, no. 48, pp. 40732-40744, 2012.

[137] Y.-J. Choi, H.-S. Shin, H. S. Choi et al., "Uric acid induces fat accumulation via generation of endoplasmic reticulum stress and SREBP-1c activation in hepatocytes," Laboratory Investigation, vol. 94, no. 10, pp. 1114-1125, 2014.

[138] Y. Y. Sautin, T. Nakagawa, S. Zharikov, and R. J. Johnson, "Adverse effects of the classic antioxidant uric acid in adipocytes: NADPH oxidase-mediated oxidative/nitrosative stress," American Journal of Physiology-Cell Physiology, vol. 293, no. 2, pp. C584-C596, 2007.

[139] W. Baldwin, S. McRae, G. Marek et al., "Hyperuricemia as a mediator of the proinflammatory endocrine imbalance in the adipose tissue in a murine model of the metabolic syndrome," Diabetes, vol. 60, no. 4, pp. 1258-1269, 2011.

[140] J. Henao-Mejia, E. Elinav, C. Jin et al., "Inflammasomemediated dysbiosis regulates progression of NAFLD and obesity," Nature, vol. 482, no. 7384, pp. 179-185, 2012.

[141] A. Wree, M. D. McGeough, C. A. Peña et al., "NLRP3 inflammasome activation is required for fibrosis development in NAFLD," Journal of Molecular Medicine, vol. 92, no. 10, pp. 1069-1082, 2014.

[142] L. J. Dixon, C. A. Flask, B. G. Papouchado, A. E. Feldstein, and L. E. Nagy, "Caspase-1 as a central regulator of high fat dietinduced non-alcoholic steatohepatitis," PLoS ONE, vol. 8, no. 2, Article ID e56100, 2013.

[143] T. Matsuzaka, A. Atsumi, R. Matsumori et al., "Elovl6 promotes nonalcoholic steatohepatitis," Hepatology, vol. 56, no. 6, pp. 2199-2208, 2012.

[144] K. Miura, L. Yang, N. van Rooijen, D. A. Brenner, H. Ohnishi, and E. Seki, "Toll-like receptor 2 and palmitic acid cooperatively contribute to the development of nonalcoholic steatohepatitis through inflammasome activation in mice," Hepatology, vol. 57, no. 2, pp. 577-589, 2013.

[145] A. Bashiri, D. Nesan, G. Tavallaee et al., "Cellular cholesterol accumulation modulates high fat high sucrose (HFHS) dietinduced ER stress and hepatic inflammasome activation in the development of non-alcoholic steatohepatitis," Biochimica et Biophysica Acta-Molecular and Cell Biology of Lipids, vol. 1861, no. 7, pp. 594-605, 2016.

[146] W. Rathmann, E. Funkhouser, A. R. Dyer, and J. M. Roseman, "Relations of hyperuricemia with the various components of the insulin resistance syndrome in young black and white adults: the CARDIA study," Annals of Epidemiology, vol. 8, no. 4, pp. 250-261, 1998.

[147] F. Facchini, Y.-D. I. Chen, C. B. Hollenbeck, and G. M. Reaven, "Relationship between resistance to insulin-mediated glucose uptake, urinary uric acid clearance, and plasma uric acid concentration," Journal of the American Medical Association, vol. 266, no. 21, pp. 3008-3011, 1991.

[148] W. Y. Tae, C. S. Ki, S. S. Hun et al., "Relationship between serum uric acid concentration and insulin resistance and metabolic syndrome," Circulation Journal, vol. 69, no. 8, pp. 928-933, 2005.

[149] E. S. Ford, C. Li, S. Cook, and H. K. Choi, "Serum concentrations of uric acid and the metabolic syndrome among US children and adolescents," Circulation, vol. 115, no. 19, pp. 25262532, 2007.

[150] T. Keenan, M. J. Blaha, K. Nasir et al., "Relation of uric acid to serum levels of high-sensitivity c-reactive protein, triglycerides, and high-density lipoprotein cholesterol and to hepatic steatosis," American Journal of Cardiology, vol. 110, no. 12, pp. 1787-1792, 2012.

[151] B. E. K. Klein, R. Klein, and K. E. Lee, "Components of the metabolic syndrome and risk of cardiovascular disease and diabetes in Beaver Dam," Diabetes Care, vol. 25, no. 10, pp. 17901794, 2002.

[152] H. Yuan, C. Yu, X. Li et al., "Serum uric acid levels and risk of metabolic syndrome: a dose-response meta-analysis of prospective studies," Journal of Clinical Endocrinology and Metabolism, vol. 100, no. 11, pp. 4198-4207, 2015.

[153] V. Bhole, J. W. J. Choi, S. Woo Kim, M. De Vera, and H. Choi, "Serum uric acid levels and the risk of type 2 diabetes: A Prospective Study," American Journal of Medicine, vol. 123, no. 10, pp. 957-961, 2010.

[154] S. Kodama, K. Saito, Y. Yachi et al., "Association between serum uric acid and development of type 2 diabetes," Diabetes Care, vol. 32, no. 9, pp. 1737-1742, 2009.

[155] Z. Jia, X. Zhang, S. Kang, and Y. Wu, "Serum uric acid levels and incidence of impaired fasting glucose and type 2 diabetes mellitus: a meta-analysis of cohort studies," Diabetes Research and Clinical Practice, vol. 101, no. 1, pp. 88-96, 2013.

[156] Q. Lv, X.-F. Meng, F.-F. He et al., "High serum uric acid and increased risk of type 2 diabetes: a systemic review and metaanalysis of prospective cohort studies," PLoS ONE, vol. 8, no. 2, Article ID e56864, 2013.

[157] R. Pfister, D. Barnes, R. Luben et al., "No evidence for a causal link between uric acid and type 2 diabetes: a mendelian randomisation approach," Diabetologia, vol. 54, no. 10, pp. 25612569, 2011.

[158] I. Sluijs, M. V. Holmes, Y. T. van der Schouw et al., "A mendelian randomization study of circulating uric acid and type 2 diabetes," Diabetes, vol. 64, no. 8, pp. 3028-3036, 2015.

[159] O. Yiginer, F. Ozcelik, T. Inanc et al., "Allopurinol improves endothelial function and reduces oxidant-inflammatory enzyme of myeloperoxidase in metabolic syndrome," Clinical Research in Cardiology, vol. 97, no. 5, pp. 334-340, 2008.

[160] E. Fabbrini, M. Serafini, I. C. Baric, S. L. Hazen, and S. Klein, "Effect of plasma uric acid on antioxidant capacity, oxidative stress, and insulin sensitivity in obese subjects," Diabetes, vol. 63, no. 3, pp. 976-981, 2014.

[161] G. van den Berghe, M. Bronfman, R. Vanneste, and H. G. Hers, "The mechanism of adenosine triphosphate depletion in the liver after a load of fructose. A kinetic study of liver adenylate deaminase," Biochemical Journal, vol. 162, no. 3, pp. 601-609, 1977.

[162] C. Cicerchi, N. Li, J. Kratzer et al., "Uric acid-dependent inhibition of AMP kinase induces hepatic glucose production in diabetes and starvation: evolutionary implications of the uricase loss in hominids," FASEB Journal, vol. 28, no. 8, pp. 3339-3350, 2014.

[163] M. A. Lanaspa, C. Cicerchi, G. Garcia et al., "Counteracting roles of AMP deaminase and AMP kinase in the development of fatty liver," PLoS ONE, vol. 7, no. 11, Article ID e48801, 2012.

[164] Y.-J. Choi, Y. Yoon, K.-Y. Lee et al., "Uric acid induces endothelial dysfunction by vascular insulin resistance associated with the impairment of nitric oxide synthesis," FASEB Journal, vol. 28, no. 7, pp. 3197-3204, 2014.

[165] Y. Zhu, Y. Hu, T. Huang et al., "High uric acid directly inhibits insulin signalling and induces insulin resistance," Biochemical and Biophysical Research Communications, vol. 447, no. 4, pp. 707-714, 2014. 
[166] H.-J. Lee, K. H. Jeong, Y. G. Kim et al., "Febuxostat ameliorates diabetic renal injury in a streptozotocin-induced diabetic rat model," American Journal of Nephrology, vol. 40, no. 1, pp. 5663, 2014.

[167] L. G. Sánchez-Lozada, E. Tapia, V. Soto et al., "Effect of febuxostat on the progression of renal disease in 5/6 nephrectomy rats with and without hyperuricemia," Nephron-Physiology, vol. 108, no. 4, pp. 69-78, 2008.

[168] H. Omori, N. Kawada, K. Inoue et al., "Use of xanthine oxidase inhibitor febuxostat inhibits renal interstitial inflammation and fibrosis in unilateral ureteral obstructive nephropathy," Clinical and Experimental Nephrology, vol. 16, no. 4, pp. 549-556, 2012.

[169] H. Tsuda, N. Kawada, J.-Y. Kaimori et al., "Febuxostat suppressed renal ischemia-reperfusion injury via reduced oxidative stress," Biochemical and Biophysical Research Communications, vol. 427, no. 2, pp. 266-272, 2012.

[170] K. Tanaka, S. Hara, A. Kushiyama et al., "Risk of macrovascular disease stratified by stage of chronic kidney disease in type 2 diabetic patients: critical level of the estimated glomerular filtration rate and the significance of hyperuricemia," Clinical and Experimental Nephrology, vol. 15, no. 3, pp. 391-397, 2011.

[171] K. Tanaka, S. Hara, M. Hattori et al., "Role of elevated serum uric acid levels at the onset of overt nephropathy in the risk for renal function decline in patients with type 2 diabetes," Journal of Diabetes Investigation, vol. 6, no. 1, pp. 98-104, 2015.

[172] K. Tanaka, M. Nakayama, M. Kanno et al., "Renoprotective effects of febuxostat in hyperuricemic patients with chronic kidney disease: a parallel-group, randomized, controlled trial," Clinical and Experimental Nephrology, vol. 19, no. 6, pp. 10441053, 2015.

[173] S. Kato, M. Ando, T. Mizukoshi et al., "Randomized control trial for the assessment of the anti-albuminuric effects of topiroxostat in hyperuricemic patients with diabetic nephropathy (the ETUDE study)," Nagoya Journal of Medical Science, vol. 78, no. 2, pp. 135-142, 2016.

[174] L. G. Sánchez-Lozada, E. Tapia, P. Bautista-García et al., "Effects of febuxostat on metabolic and renal alterations in rats with fructose-induced metabolic syndrome," American Journal of Physiology-Renal Physiology, vol. 294, no. 4, pp. F710-F718, 2008.

[175] C. Xu, X. Wan, L. Xu et al., "Xanthine oxidase in non-alcoholic fatty liver disease and hyperuricemia: one stone hits two birds," Journal of Hepatology, vol. 62, no. 6, pp. 1412-1419, 2015.

[176] K. Schröder, C. Vecchione, O. Jung et al., "Xanthine oxidase inhibitor tungsten prevents the development of atherosclerosis in ApoE knockout mice fed a Western-type diet," Free Radical Biology and Medicine, vol. 41, no. 9, pp. 1353-1360, 2006.

[177] K. G. Soucy, H. K. Lim, D. O. Attarzadeh et al., "Dietary inhibition of xanthine oxidase attenuates radiation-induced endothelial dysfunction in rat aorta," Journal of Applied Physiology, vol. 108, no. 5, pp. 1250-1258, 2010.

[178] R. P. Jankov, C. Kantores, J. Pan, and J. Belik, "Contribution of xanthine oxidase-derived superoxide to chronic hypoxic pulmonary hypertension in neonatal rats," American Journal of Physiology_Lung Cellular and Molecular Physiology, vol. 294, no. 2, pp. L233-L245, 2008.

[179] A. N. Shafik, "Febuxostat improves the local and remote organ changes induced by intestinal ischemia/reperfusion in rats," Digestive Diseases and Sciences, vol. 58, no. 3, pp. 650-659, 2013.

[180] A. Patt, A. H. harken, L. K. Burton et al., "Xanthine oxidase-derived hydrogen peroxide contributes to ischemia reperfusion-induced edema in gerbil brains," The Journal of Clinical Investigation, vol. 81, no. 5, pp. 1556-1562, 1988.

[181] A. Kumar, G. Bhawani, N. Kumari, K. S. N. Murthy, V. Lalwani, and C. N. Raju, "Comparative study of renal protective effects of allopurinol and n-acetyl-cysteine on contrast induced nephropathy in patients undergoing cardiac catheterization," Journal of Clinical and Diagnostic Research, vol. 8, no. 12, pp. HC03-HC07, 2014.

[182] S. Wang, Y. Li, X. Song et al., "Febuxostat pretreatment attenuates myocardial ischemia/reperfusion injury via mitochondrial apoptosis," Journal of Translational Medicine, vol. 13, no. 1, article 209, 2015.

[183] M. Duda, A. Konior, E. Klemenska, and A. Beresewicz, "Preconditioning protects endothelium by preventing ET-1-induced activation of NADPH oxidase and xanthine oxidase in postischemic heart," Journal of Molecular and Cellular Cardiology, vol. 42, no. 2, pp. 400-410, 2007. 


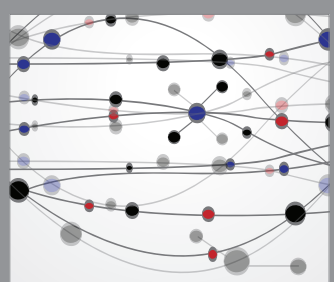

The Scientific World Journal
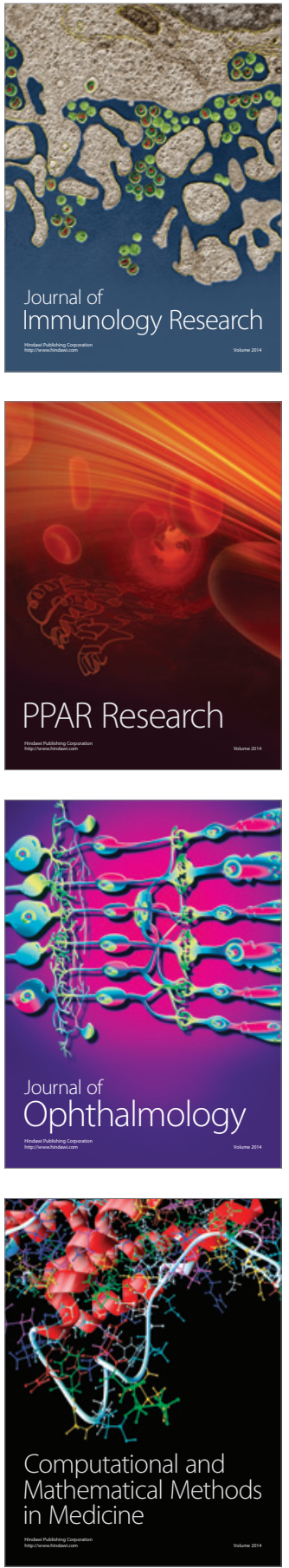

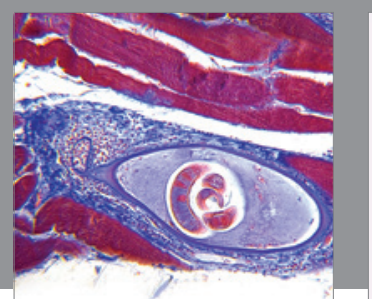

Gastroenterology Research and Practice

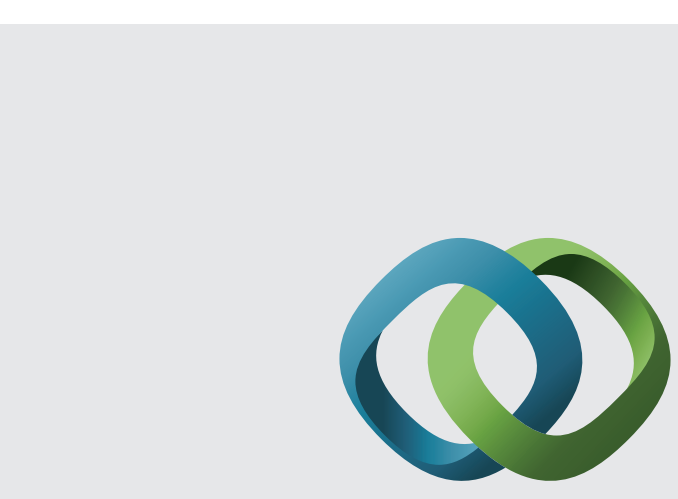

\section{Hindawi}

Submit your manuscripts at

http://www.hindawi.com
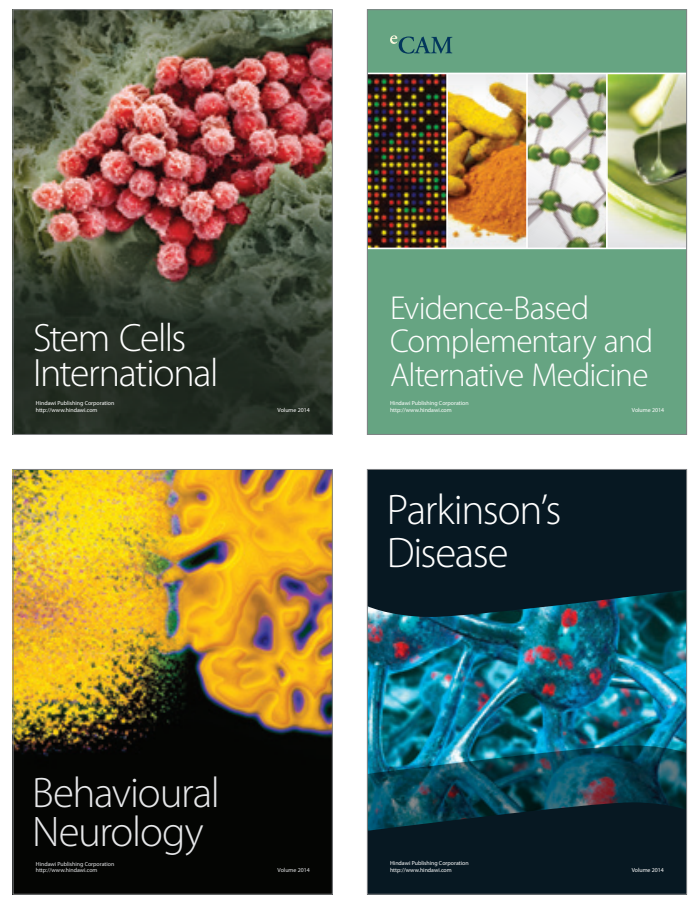
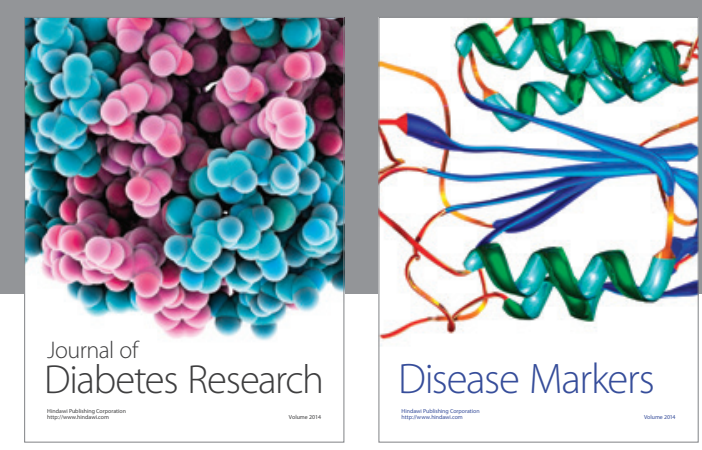

Disease Markers
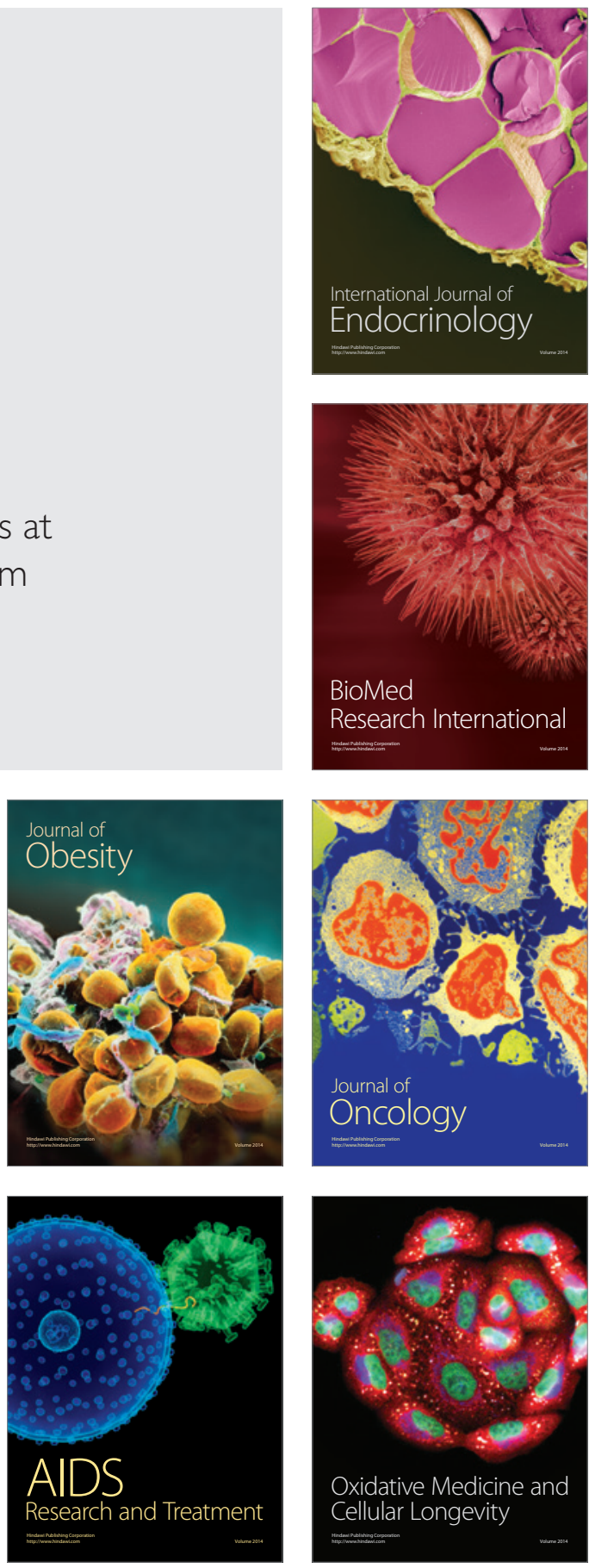